MANUEL FERNANDO QUEIROZ DOS SANTOS JUNIOR

\title{
ASPECTOS DO CANCRO MOLE NO \\ MUNICÍPIO DE SÃO PAULO
}

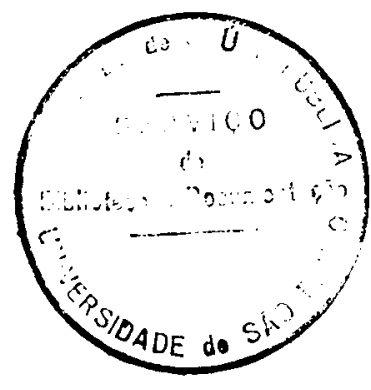

Tese de doutoramento apresentada ao Departamento de Epidemiologia da Faculdade de Saúde Pública da Universidade de São Paulo Orientador:

Profe Dre Oswaldo Paulo Forattini

São Paulo 
Aos meus pais: Marina Alvacoeli e Manuel Fernando

e minhas irmãs: Marina e Ana Maria pelo estímulo. 
A Walter Belda, mestre, colega e amigo precocemente desaparecido a quem devo os primeiros passos pelos caminhos da Saúde Pública. 
A Altino Cattapan, venereologista, amigo, exemplo de sabedoria. 


\section{AGRADECIMENTOS}

A todas as pessoas que direta ou indiretamente colaboraram para que se tornasse possível a realização deste trabalho, em especial.

ao Prof Luiz Fernando de Góes Siqueira

Profo Walter Belda Junior

à Srä Alayde Siqueira Marcondes

Sra Angela Maria Belloni Cuenca

Sra Cleide Favilla

Sro Cícero Souza de Oliveira

Sra Elaine Cristina Barrelo Oliveira

Sra Glei Marlene da Silva

Enfa Luciana Ferreira Xavier Junqueira

Sr Lucio Simphronio

Sra Maridalva Toso Barberi

Srtª Maria de Jesus dos Santos

Sre Nelson Barrelo Junior

Sr Pedro Manoel dos Santos

Sra Regina Gomes de Almeida

Enfa Tania Eloise Lafrata 


\section{RESUMO}

No período compreendido entre 1985 a 1989 foram registrados no Serviço de Dermatologia Sanitária da Faculdade de Saúde Pública de Universidade de São Paulo 202 pacientes portadores de cancro mole, o que compreendeu o universo de estudo.

Mapeou-se os possiveis focos geográficos da doençe distribuídos pelos 11 Distritos, 48 Subdistritos e 8 Escritórios Regionais de Saúde (ERSA) que compõe o Município de São Paulo. Os subdistritos do Butantã e Santo Amaro concentraram o maior número de casos (55) e o ERSA-1 apresentou 74 casos (36,6\%).

A idade concentrou-se entre 20 e 30 anos, havendo um aumento progressivo em menores de 20 anos. Os homens participaram numa proporção de 7:1 mulher, com tendência a diminuir. Houve nítida redução da participação da prostituição como fonte de contágio.

O período de incubação variou de 1 a 41 dias com 60,5\% nos primeiros 7 dias. A lesão foi única em $33,7 \%$ dos casos masculinos. $O$ tempo da doença reduziu-se e o enfartamento inguinal ocorreu em $49 \%$ dos casos. Referiram passado venéreo 123 pacientes variando de 1 a mais de 5 episódios.

A soropositividade para sífilis revelou-se alta $(28,2 \%)$.

Em um outro período (1989-1991) foram pesquisados 42 casos de cancro mole para Anticorpos anti-Vírus da Imunodeficiência Humana 
(VIH-1) confirmados pelo WESTERN BLOT, resultando $16,6 \%$ de sororeatividade. O estudo desta soropositividade revelou que o risco da mulher portadora de um cancro mole apresentar-se infectada pelo $\mathrm{VIH}-1$ parece ser maior do que para o homem.

Diante destes resultados, recomenda-se a implantação imediata da notificação compulsória de todas as Doenças Sexualmente Transmissíveis, bem como a pesquisa rotineira para sifilis e para o $\mathrm{VIH}-1$ nas populações de risco identificadas. 


\section{S U M M AR Y}

Two-hundred-and-two chancroidal patients registered at the Sanitarian Dermatological Service of the University of São Paulo School of Public Health in São Paulo city, State of São Paulo, Brazil, within the period from 1985 to 1989, comprehended our universe of study. Possible geographical focuses of the disease, distributed among the eleven districts, forty-eight subdistricts and eight Regional Health Offices (ERSAs) which make up the São Paulo municipality, were mapped out. The Butantan and Santo Amaro subdistricts were the ones in which the greatest number of cases were concentrated (55\%); ERSA-1 presented 74 cases (36.6\%). In what age is concerned to, the age group 20-30 showed the highest concentration of cases, indicating a progressive increase in individuals below 20. Male patients participated in a proportion of 7:1 in relation to women, with a diminishing tendency in the period. There was a marked reduction of prostitution as a source of contagion. The incubation period varied from 1 to 41 days, with $69.5 \%$ of the cases occuring in the first seven days. Single-lesion was presented in $33.7 \%$ of the male cases. The length of the disease were reduced in the period studied. Inguinal infarction occured in $49 \%$ of the cases. One-hundred and twenty-three patients referred venereal past, varying from one to more than five episodes. Serological tests for syphilis showed a high percentage (28.2\%).

In another period (1989-1991), 42 cases of chancroid were searched for antiviral antibodies of human immunodeficiency virus (HIV-1), 
supported by the Western-Blot, resulting in $16.6 \%$ of seroactivity. The study of this seroactivity indicated that the risk for a woman with chancroid to present herself infected by the HIV-1 appears to be greater than the one for men.

In face of these data, the immediate implantation of a compulsory notification programme regarding all sexually transmitted diseases, as well as a routine search for syphilis and HIV-1 in identified riskpopulations, are recommended. 


\section{ÍNDICE}

1.1 - Generalidades 2

1.2 - Quadro Clínico 4

1.3 - Diagnóstico 5

1.4 - Quadro Epidemiológico 6

1.5 - Prevenção 6

2 - OBJETIVOS 9

$\begin{array}{ll}3 \text { - JUSTIFICATIVA } & 11\end{array}$

4 - MATERIAL E MÉTODO 15

5 - RESULTADOS E DISCUSSÃO 19

6 - CONCLUSÕES 58

7 - REFERÊNCIAS BIBLIOGRÁFICAS 61 
Faculdade de Saúde Pública da Universidade de São Paulo, por distrito de residência, 1985-1989.

Tabela 7 - Distribuicão dos diagnósticos de Cancro Mole realizados no Município de São Paulo - Serviço de Dermatologia Sanitária da Faculdade de Saúde Pública da Universidade de São Paulo, por Escritório Regional de Saúde (ERSA), 1985-1989.

Tabela 8 - Distribuição dos Distritos e Subdistritos do Município de São Paulo, por Escritório Regional de Saúde.

Tabela 9 - Distribuição dos diagnósticos de Cancro Mole realizados no Município de São Paulo - Serviço de Dermatologia Sanitária da Faculdade de Saúde Pública da Universidade de São Paulo, por hábito sexual, 1985-1989.

Tabela 10 - Distribuição dos diagnósticos de Cancro Mole realizados no Município de São Paulo - Serviço de Dermatologia Sanitária da Facúldade de Saúde Pública da Universidade de São Paulo, por idade do início sexual, 1985-1989.

Tabela 11 - Distribuição dos diagnósticos de Cancro Mole realizados no Município de São Paulo - Serviço de Dermatologia Sanitária da Faculdade de Saúde Pública da Universidade de São Paulo, por número pregresso de episódios venéreos, 1985-1989.

Tabela 12 - Distribuição dos diagnósticos de Cancro Mole realizados no Município de São Paulo - Serviço de Dermatologia Sanitária da Faculdade de Saúde Pública da Universidade de São Paulo, por diagnóstico do primeiro episódio venéreo, 1985-1989.

Tabela 13 - Distribuição dos diagnósticos de Cancro Mole realizados no Município de São Paulo - Serviço de Dermatologia Sanitária da 
Faculdade de Saúde Pública da Universidade de São Paulo, por faixa etária do primeiro episódio venéreo, 1985-1989.

Tabela 14 - Distribuição dos diagnósticos de Cancro Mole realizados no Município de São Paulo - Serviço de Dermatologia Sanitária da Faculdade de Saúde Pública da Universidade de São Paulo, por tipo fonte, 1985-1989.

Tabela 15 Distribuição dos diagnósticos de Cancro Mole realizados no Município de São Paulo - Serviço de Dermatologia Sanitária da Faculdade de Saúde Pública da Universidade de São Paulo, por período de incubação, 1985-1989.

Tabela 16 - Distribuição dos diagnósticos de Cancro Mole realizados no Município de São Paulo - Serviço de Dermatologia Sanitária da Faculdade de Saúde Pública da Universidade de São Paulo, por tempo de doença, 1985-1989.

Tabela 17 - Distribuição dos diagnósticos de Cancro Mole realizados no Município de São Paulo - Serviço de Dermatologia Sanitária da Faculdade de Saúde Pública da Universidade de São Paulo, por número de lesões, 1985-1989.

Tabela 18 - Distribuição dos diagnósticos de Cancro Mole realizados no Município de São Paulo - Serviço de Dermatologia Sanitária da Faculdade de Saúde Pública da Universidade de São Paulo, por enfartamento inguinal, 1985-1989.

Tabela 19 - Distribuição dos diagnósticos de Cancro Mole realizados no Município de São Paulo - Serviço de Dermatologia Sanitária da Faculdade de Saúde Pública da Universidade de São Paulo, por resultado qualitativo do VDRL, 1985-1989. 
Tabela 20 - Distribuição dos diagnósticos de Cancro Mole realizados no Município de São Paulo - Serviço de Dermatologia Sanitária da Faculdade de Saúde Pública da Universidade de São Paulo, por resultado quantitativo do VDRL, 1985-1989.

Tabela 21 - Distribuição dos diagnósticos de Cancro Mole realizados no Município de São Paulo - Serviço de Dermatologia Sanitária da Faculdade de Saúde Pública da Universidade de São Paulo, por resultado de deteç̧ão de Anticorpos Anti-HIV-1 e WESTERN BLOT, 1989-1991.

Tabela 22 - Distribuição dos diagnósticos de Cancro Mole realizados no Município de São Paulo - Serviço de Dermatologia Sanitária da Faculdade de Saúde Pública da Universidade de São Paulo, por resultado do teste de ELISA e WESTERN BLOT, e sexo, 19891991.

Figura 1 - Ficha Clínico-epidemiológica.

Figura 2 - Distribuição dos Distritos e Subdistritos do Municipio de São Paulo.

Figura 3 - Distribuição dos Escritórios Regionais de Saúde do Município de São Paulo. 


\section{1 - INTRODUÇÃO}

\section{1 - Generalidades}

Embora de etiologias distintas, as chamadas "Doenças Sexualmente Transmissiveis" (DST) constituem grupo de infecções que têm, em comum quadros epidemiológicos similares. Nos últimos decênios têm-se acumulado dados sobre essas afecções e, com o advento da síndrome da imunodeficiência adquirida (SIDA/AIDS), incrementou-se a conscientização populacional a respeito das DST e dos riscos da prática sexual promíscua. Em relação às doenças tradicionalmente conhecidas, deu-se também a aquisição de novos conhecimentos como, por exemplo, no que concerne à associação do câncer do colo do útero e das infecções por vírus do papilona. Não obstante, os aspectos epidemiológicos das DST não são totalmente conhecidos, em relação a múltiplas feições. Pode-se dizer que estas variam no tempo e no espaço, obedecendo a fatores dificilmente contornáveis. Entre estes, pode-se mencionar a subnotificação e a variabilidade histórica do comportamento sexual.

A essa feição geral, não constitui exceção o Cancro Mole, também conhecido como úlcera venérea. Sua associação com baixos níveis sócioeconômicos e educacionais é sobejamente conhecida (GAY PRIETO, 1966; BELDA, 1982). 
Bem assim como sua variação espacial como, por exemplo, nos EUA onde é geralmente raro, mas tão comum quanto a gonorréia em certas áreas do leste desse país (SPARLING, 1992).

Com a introdução da penicilinoterapia e o controle rigoroso da prostituição nos anos 50 , os dispensários anti-venéreos com seu papel cumprido, passam a ser desativados, erro decorrente da interpretação da curva epidemiológica de países industrializados e que motivou o comitê de expertos da O.M.S. a declarar a erradicação das DST para o final da década de 50. No entanto, com o início sexual precoce masculino e feminino, ocorre a expansão da sexualidade e com ela a promiscuidade, isto é, a aceitação consentida da variação de parceiros em intensidade e freqüência. Em decorrência, à faixa dos 20 aos 30 anos acrescentou-se a dos adolescentes. Ao relacionamento amoroso associa-se gama de variações, propiciando não só a colonização de agentes patógenos nas outras mucosas, mas fundamentalmente facilitando novas vias de transmissão.

Ampliou-se, com isso o arsenal de patógenos imputados de transmissão sexual, hoje cerca de 30 agentes.

Recentemente, tem-se intensificado estudos de modelos matemáticos objetivando melhor entendimento dos aspectos epidemiológicos das DST. Para tanto têm sido valioso a observação desse tipo de afecções em populações de animais domésticos (SMITH E DOBSON 1992). 


\section{2 - Quadro Clínico}

Esta afecção pode ser definida como infecção cujo agente etiológico é o bacilo gram-negativo Haemophilus ducreyi; e que, no âmbito das DST enquadra-se como "doença essencialmente transmitida por contato sexual" (PEREIRA JR. \& SERRUYA, 1981).

Caracterizada por úlceras necróticas, dolorosas, cobertas por secreção purulenta que exala um odor fétido, é de localização predominantemente genital, podendo atingir a região genito-anal ou anal. Por vezes única é acompanhada ou não de linfadenopatia satélite inguinal uni ou bilateral (RABELO, 1980).

Raramente atinge localização extragenital. Recentemente foi reportado um caso no pé sem história clínica de infecção primária genital. (CASTRO et al 1937; BORGES 1938; MARCKMAN et al 1989).

São recentes as observações de ABECK \& KORTING (1991) a cerca do mecanismo de aderência, penetração e produção de necrose do tecido pelo $\underline{H}$. ducreyi.

O período de incubação mais freqüente ocorre dentro da primeira semana, em média 2 a 5 dias após o contato sexual suspeito, não havendo sinais prodrômicos ou sintomas.

A lesão ulcerosa aparece inicialmente como pápula com eritema marginal. O centro se pustula e rapidamente erosa formando úlcera em 48/72 horas. Não há induração como no cancro primário da sífilis, sendo por isso chamado de cancro mole. A base da úlcera aparece suja e necrótica, podendo haver infecção secundária exalando odor fétido 
característico. Devido à autoinoculação as lesões podem ser múltiplas "espírito de família" - RICORD (1838) e coalescer, formando úlceras gigantes com destruição de tecido e edema. O caráter doloroso da lesão, constante e intenso, é fator importante para o diagnóstico. (SERRUYA, 1977; RUDOLPH, 1987).

A linfoadenopatia inguinal, uni ou bilateral (bubões), pode ocorrer e evoluir, quando não tratada, para supuração por um único orifício.

O cancro diminuto por vezes com aspecto de foliculite pode ser confundido com herpes genital, já o cancro gigante é passível de ser confundido com a donovanose.

A associação com Treponema pallidum causa o cancro misto ou cancro de ROLLET, pouco encontrado no nosso meio.

Quando involui, o cancro mole pode deixar cicatriz e a recorrência é freqüente.

\section{3 - Diagnóstico}

Na prática recomenda-se não limpar a lesão, coletar o material purulento da lesão ou lesões com a alça de platina, preparando um esfregaço uniforme, efetuar a coloração de GRAM utilizando-se safranina para melhor resolução das paredes do $\underline{H}$. ducreyi.

No nosso meio a visualização dos bacilos gram negativos intracelulares associados a cocos gram positivos é indicador identificação do agente etiológico. 


\section{4 - Quadro Epidemiológico}

Nesta doença a infectividade e a patogenicidade são altas, o que Ihe dá caráter de elevada contagiosidade. A virulência é baixíssima, devido ao fato das lesões atingirem apenas o tegumento, não sendo descritas alterações sistêmicas. Tais feições muito contribuem para as imprecisões que cercam o diagnóstico dessa doença.

Desconhece-se o quadro epidemiológico dessa doença em nossa nosologia. Em se tratando de afecção de baixa virulência e para a qual inexiste notificação compulsória, entende-se as dificuldades que daí resultam. É bem verdade que têm-se registrado surtos da doença em outras regiões (LYKKE-OLESEN e col. 1979; HAMMONED e col. 1980; BLACKMORE e col. 1985). No entanto, tais fenômenos ainda não foram assinalados em nosso meio. Compreende-se pois as dificuldades que advêm para a descrição do quadro epidemiológico. Acresce considerar, como já se referiu, a variabilidade de sua incidência e prevalência, sujeitas que estão à influência de determinantes sociais de caráter local (FORATTINI, 1992).

\section{5 - Prevenção}

Para os países latino-americanos existe opinião unânime acerca da necessidade da integração nos serviços gerais de saúde pública. Integração multiprofissional e multidisciplinar devendo haver para isso uma 
estreita relação entre a dermatologia sanitária com as clínicas universitárias (SANTOS JR., 1986).

Torna-se necessário o estabelecimento de programa específico, até então discutido e não implantado. Recomenda-se a implantação definitiva de sistema de notificação compulsória das DST para determinar a análise real da situação e conseqüentes medidas de profilaxia.

Mas não existe ainda essa notificação ampla, restrita que está à síndrome da imunodeficiência adquirida (SIDA/AIDS) e à sífilis congênita (MINISTÉRIO DA SAÚDE, 1986). Localmente, essa medida foi estendida à sífilis congênita no Estado de São Paulo (SECRETARIA DA SAÚDE DE SÃO PAULO, 1990).

O panorama populacional das DST em nosso meio tem recebido apenas extensões individualizadas, tal é o caso no Estado do Mato Grosso do Sul, onde através de aerograma conseguiu-se mapear o problema das DST no Estado. Intensifica-se a necessidade do ensino específico em todos os níveis. (SECRETARIA DA SAÚDE DO ESTADO DE MATO GROSSO DO SUL, 1992). Não obstante, freqüentemente analisa-se a ignorância sobre esse tema, por parte dos profissionais (SIBOULET 1975; BELDA, 1983).

Torna-se pois necessário intensificar o ensino e treinamento sobre DST nos cursos universitários, aliado a cursos de atualização freqüentes.

A necessidade estratégica básica de intervenção na cadeia de transmissão está no chamado pronto atendimento, mecanismo simples que impede que a fonte permaneça infectante, somado à busca ativa de contatos. O atendimento realizado em farmácias, onde o "farmacista", isto é 
o balconista, resolve o problema rapidamente aplicando a "empurroterapia", encorajou o estabelecimento, no Serviço de Dermatologia Sanitária da Faculdade de Saúde Pública da Universidade de São Paulo, de dinâmica de atendimento rápido, associado a pequeno laboratório. (BELDA, 1982). 


\section{2 - OBJETIVOS}




\section{2 - OBJETIVOS}

Face as circustâncias explanadas nos parágrafos anteriores, as feições epidemiológicas do cancro mole somente poderão ser determinadas de maneira indireta, uma delas poderá basear-se no impacto que a doença apresenta nos serviços assistenciais, de atendimento ambulatorial à população. A outra seria a de associá-la a outra DST, mas de notificação compulsória, e avaliar a sua distribuição relativa aos vários aspectos do quadro epidemiológico. Ambos os procedimentos foram levados a efeito neste estudo, cujos resultados constituem o objeto da presente tese. 


\section{3 - JUSTIFICATIVA}

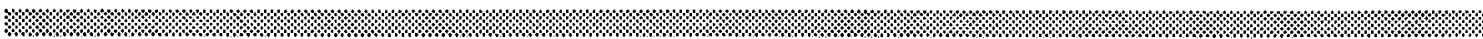
w 


\section{3 - JUSTIFICATIVA}

É intenção nossa oferecer parcela de colaboração para o entendimento da situação de uma das doenças sexualmente transmissiveis ulcerativas no município de São Paulo.

Sendo o cancro mole doença de incidência universal e considerada "a mais venérea das doenças venéreas" (GAY-PRIETO 1966), com freqüência ligada as baixas condições sócio econômicas, quase desapareceu nos anos que sucederam a segunda guerra mundial.

No início dos anos 70 houve um aparente reaparecimento detectado no Brasil no Rio Grande do Sul (BOPP et al - 1973 ), na França (DUPERRAT et al - 1973; MOREL - 1974; SABLET \& PUISSANT - 1974) e posteriormente na América, África e Ásia (MINDEL - 1989).

Em nosso meio, BELDA (1983) já alertava sobre a elevação de casos registrados em São Paulo tendo nos estimulado sobre o tema e, sugerido a realização do estudo durante o quinqũênio 1985 a 1989, devido a tendência de recrudescimento.

Das 650 referências bibliográficas levantadas de 1889 a 1991, 5\% referem-se a estudos epidemiológicos mundiais. Foram 33 trabalhos nacionais, sendo o de OLIVEIRA (1951), o único estudo epidemiológico.

A abordagem da epidemiologia do cancro mole torna-se justificável e mesmo imperativa devido a:

a) sua possivel potencialidade como problema de saúde

pública 
b) a inexistência do conhecimento epidemiológico em nosso meio

c) seu possivel caráter indicativo de determinantes sociais das DST

d) a possibilidade da obtenção de subsídios úteis para a formulação mais segura e avaliação mais adequada dos programas de controle.

A não existência de notificação das D S T. e a conseqüente dificuldade de informação sobre o problema, levou-nos a pensar ser oportuno a realização deste trabalho para divulgar o seu possível impacto sobre as atividades de assistência médica. Em decorrência, os conhecimentos ambulatoriais do serviço de dermatologia sanitária do Centro de Saúde "Geraldo Horácio de Paula Souza" da Faculdade de Saúde Pública da Universidade de São Paulo, afiguram-se bastante propícios. E isso por várias razões, dentre as quais o de ser herdeiro do antigo serviço antivenéreo do Instituto de Higiene instalado em 1924, e assim constituindo-se em polo de drenagem natural de pacientes dos distritos e sub-distritos do município e da grande São Paulo. Além disso mantém-se como local de treinamento e de pesquisa, além de assim fornecer indícios que poderiam possibilitar o levantamento de hipóteses sobre a eventual população de risco. Desta forma estaríamos contribuindo para futuros inquéritos epidemiológicos diretos.

Sendo o cancro mole uma das ulcerações genitais e a evidência da associação entre as ulceras genitais e o vírus da imunodeficiência humana-1 (VIH-1) (GREENBLATT et al - 1988; KREISS et al - 1989; PLUMMER et al - 1990) levou-nos a incluir a pesquisa de 
anticorpos anti-VIH-1 nos portadores de Cancro Mole, na tentativa de identificar algumas possíveis características populacionais em nosso meio.

Utilizamos período de 1989 a 1991 devido ao fato do Laboratório de Dermatologia Sanitária ter iniciado nessa data, a rotina de pesquisa de anticorpos anti VIH-1. 


\section{4 - MATERIAL E MÉTODO}

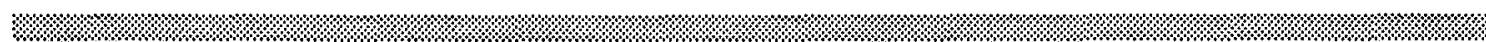
\% 


\section{4 - MATERIAL E MÉTODO}

Neste estudo analisou-se, ano a ano, 202 pacientes consecutivos, portadores de cancro mole que, como se referiu, procuraram espontâneamente o Serviço de Dermatologia Sanitária do Centro de Saúde Escola "Geraldo Horácio de Paula Souza" da Faculdade de Saúde Pública da Universidade de São Paulo, no quinqüênio 1985-1989.

Em etapa inicial, estabelecido o diagnóstico clínico e confirmado o laboratorialmente, procedeu-se pessoalmente o preenchimento da Ficha Clínico Epidemiológica implantada em 1984. (SANTOS JR., 1986) (figura 1), quanto aos atributos:

1 - Pessoais

Idade

Sexo

Raça

Estado Civil

Escolaridade

Local de Residência

2 - Comportamentais

Idade do início sexual

Hábito sexual predominante

Primeiro acidente venéreo

Idade do primeiro acidente venéreo

Passado venéreo 
3 - Clínicos da Doença Atual

Tipo do fonte

Período de incubação

Tempo de doença

Número de lesões

Presença de enfartamento ingüinal

4 - Laboratoriais

Reatividade ao VDRL e FTA abs

Estes atributos foram tabulados manualmente ano a ano segundo os diferentes ítens, além da distribuição pelos distritos e subdistritos de residência e por Escritório Regional de Saúde (ERSA) do Município de São Paulo.

Do ponto de vista laboratorial, foram coletados $5 \mathrm{ml}$ de sangue para a realização da sorologia cardiolipinica para Sífilis (NDRL) e sorologia treponêmica (F T A - Abs) confirmatória, de todos os pacientes incluídos.

No final do ano de 1988 o laboratório de Dermatologia Sanitária passou a realizar como rotina a pesquisa de Anticorpos anti vírus da imunodeficiência humana-1, através do exame de triagem ELISA que quando positivo era repetido e se permanecesse positivo era feito o exame confirmatório de WESTERN-BLOT.

Durante o período 1989 a 1991 os resultados obtidos foram consolidados e tabulados. 
ficuian 1: Ficha clinico - EpICantolcerca

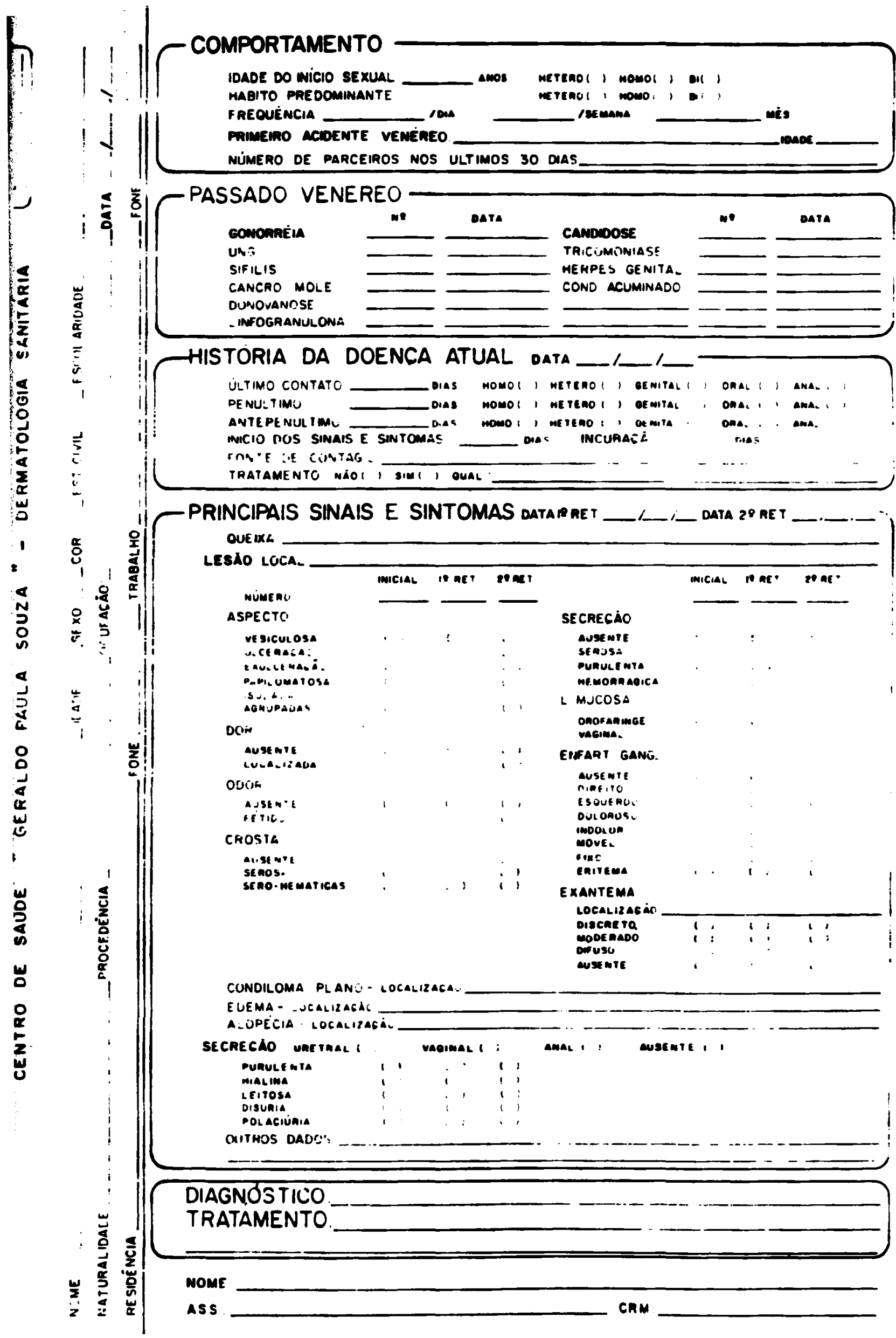




\section{5 - RESULTADOS E DISCUSSÃO}




\section{5 - RESULTADOS E DISCUSSÃO}

Do ponto de vista quantitativo, o cancro mole parece apresentar uma trajetória universal inconstante. Durante os anos de 1924 e 1925 o Instituto de Higiene registrou apenas 1 caso de cancro mole (LEME 1926). A Clínica de Dermatologia-Sifiligráfica da Faculdade de Medicina de São Paulo, registra 674 casos de cancro mole no período de 1944 a 1945 (SAMPAIO, 1957). O ano de 1973 parece marcar a reaparição do cancro mole após 20 anos de virtual extinção no Brasil (BOPP - 1973), na França, (DEGOS et al - 1973; DUPERAT et al- 1973: LEPOR \& PEROL - 1973: MOREL - 1974), e na Argentina (CASAS et al - 1973).

Sob o ponto de vista epidemiológico, pode-se mencionar que, entre 1977 e 1979, na Groenlandia, registrou-se surto com 975 casos (LYKKE - OLESEN - 1979) e, em Rotterdan-Holanda, outro com 53 casos (NAYAR et al - 1979). Na África onde o cancro mole era ulceração genital não habitual (MARILL et al - 1970, BOUDGHENE - 1989), NSANZE et al (1981) registra, em Kenia 18.245 casos e MARCHAND et al (1978), em Dakar, 1.585 casos. Na Ásia, foram estudados 500 casos (TAN et al - 1977) e na Turquia MURAT et al (1978) refere um surto de 50 casos. Apesar de SATULSKY (1945) registrar 1.555 casos e ASIN (1952) 1.402 casos no dispensário geral do Exército, os maiores surtos registrados nos Estados Unidos ocorrerram em Atlanta em 1977, na cidade de New York em 1980, Flórida e Califórnia em 1981, Massachussets em 1985 e Dallas entre 1986 e 1987 - (BLACKMORE et al, 1985; CDC 1985; BECKER et al, 1987; SCHIMID et al, 1987; McCARLEY et al - 1988). 
O Centro para Controle de Doenças (CDC) de Atlanta, Estados Unidos, registra 3.405 casos em 1986, 4.986 casos em 1987, 4.891 casos em 1988, 4.697 casos em 1989 e uma concentração na cidade de New York de $61,2 \%$ dos casos registrados entre 1986 e 1989 (CDC, STD Surveillance 1990).

Em outras partes, pode-se mencionar a Espanha onde a maioria dos poucos casos registrados, na década de 80 , foram importados, sendo 2 casos autoctones (SANCHES et al, 1989). Na Inglaterra, o cancro mole é raro, apesar de surtos em países desenvolvidos (MINDEL, 1989). Por outro lado o editorial do LANCET (1982) refere que "a incidência de Cancro Mole há muito excede a da sífilis" e a Organização Mundial da Saúde prevê 2,5 milhões de casos do Cancro Mole para 1990 (WHO - 1990).

Percebemos que, apesar de alguns registros, em nosso meio pouco ou quase nada pôde ser observado. Como já nos referimos anteriormente, esse fato possa estar relacionado com a não notificação obrigatória desta afecção.

No período de 1980 a 1984 nos registros do Serviço de Dermatologia Sanitária foi possível observar crescimento crescente chegando a aumentar 500\% (BELDA et al, 1984).

Na Tabela 1 podemos observar 0 atributo idade com uma maior concentração na faixa compreendida entre 15 a 30 anos e uma variação de 64\% em 1988 a 95\% em 1989.

De maneira geral, esses dados sugerem que a afecção está atingindo um grupo mais jovem, visto que $13,9 \%$ dos casos ocorrerram entre 15 e 19 anos com uma variação de 6,3\% em 1985 para 30\% em 1989. A idade mínima observada foi de 15 anos e a máxima 73 anos. 
Estes dados são concordantes com BELDA et al (1984) que obteve $23,4 \%$ de concentração entre adolescentes e com ORELLANA-DIAZ (1988) que obteve idade mínima de 13 anos.

No Brasil a variação foi dos 11 aos 65 anos (BOPP, 1973), na África, onde o cancro mole é a D.S.T. mais freqüente, a variação foi dos 20 aos 30 anos (JOSHI - 1983) e em Paris, onde quase todos os casos são importados, a variação foi dos 16 aos 69 anos (CIVATTE, 1980).

JESSAMINE et al (1987) e CHOUINARD (1989) referem que em Winnepg a idade variou de 20 a 71 anos.

Portanto, o que observamos é que o cancro mole como todas as Doenças Sexualmente Transmissiveis atinge nesta população a mesma faixa, que vai da adolescência ao adulto jovem.

$\mathrm{Na}$ Tabela 2 observamos que $88,1 \%$ dos 220 pacientes estudados ocorreram no sexo masculino, uma proporção aproximada de 7 para 1 caso do sexo feminino. Para o ano de 1986 alcançou uma proporção de 26 para 1.

Há, portanto, indicativos de predominância nítida dos casos masculinos. BOPP (1973) faz referência a apenas 2 mulheres em 372 pacientes. MOREL et al (1982) nos 678 casos observados em Paris encontram $97,4 \%$ de pacientes masculinos. No entanto, JESSAMINE et al (1988) aponta 2:1, e BOGAERTS et al (1989) em Rwanda 1:1. No surto de Winnipeg a proporção foi de 3 homens para 1 mulher (CHOUINARD - 1989).

Esta proporção é habitualmente atribuída a fatores sociais e culturais que levariam a uma maior exposição do homem não só ao risco de contágio como também ao diagnóstico da doença. 
Como já referimos o Haemophilus ducreyi necessita de uma solução de continuidade da mucosa para poder estabelecer a doença e o papel da imunidade relacionada com a suceptibilidade ou com a patogenese. não é ainda conhecido (RONALD \& PLUMMER, 1989).

Na Tabela 3 é apresentada a distribuição por grupo étnico, tributo díficil de ser observado. Como todos os pacientes foram por nós atendidos e valendo-nos dos mesmos critérios, optamos em dividir em raça branca, negra, mulata e amarela.

Houve uma predominância em brancos com 60,9\% e uma variação de 45\% em 1989 a 69,2\% em 1987, seguida pela raça negra em 15,8\% e uma variação de $7,7 \%$ em 1987 a 25\% em 1989.

Apesar de não ser uma raça, mas produto da mistura entre brancos e negros, o mulato, de um modo geral apresentou taxas superiores aos da raça negra e inferiores da raça branca, variando de $17,5 \%$ dos casos em 1985 a $30,0 \%$ em 1989 e no total $21,8 \%$.

Nosso resultados contrastam com MAUFF et al (1983) e BECKER et al (1987) onde há nítida predominância da raça negra.

Devido a fatores culturais, 0 japoneses não costumam procurar serviços que não tenham orientação de profissionais da mesma origem, os 3 pacientes observados eram da $2^{\mathrm{a}}$ geração de japoneses e colaboraram com $1,5 \%$ do total dos casos.

Apesar de relação entre grupo étnico e nivel sócioeconômico, a raça branca foi a mais freqüente, não foi possivel estabelecer correlação com escolaridade e o tipo de profissão. 
Na Tabela 4 podemos observar a distribuição por estado civil.

Este atributo devido as modificações de comportamento estabelecidas pela revolução sexual, por vezes perde o seu papel de limitador da promiscuidade.

Optamos incluir solteiro, amasio, casado, desquitado ou separado e viúvo. O estado de amasio foi incluido para distinguir do solteiro, que apesar de não ter casado oficialmente mora com parceira fixa. Como era de se esperar houve uma predominância de solteiros $(79,7 \%)$ e os desquitados, separados oficialmente ou não há mais de um ano colaboraram com apenas 3 casos $(1,5 \%)$.

Na Tabela 5 podemos observar que $60,4 \%$ dos pacientes apresentavam pelo menos o $1^{2}$ grau incompleto e que $4,5 \%$ eram analfabetos com uma variação anual crescente de 1,6\% em 1985 para 10,0\% em 1989. É interessante notar que $8,9 \%$ dos casos apresentavam o curso superior completo.

A escolaridade, de maneira grosseira oferece pistas do nivel sócio-econômico que a nosso ver continua baixo. Outro parâmetro que pode auxiliar no estudo sócioeconômico é o local de residência.

$\mathrm{Na}$ tentativa de mapear os casos de cancro mole por local de residência, na tabela 6 procurou-se distribuir pelos 11 Distritos e 48 Subdistritos do Município de São Paulo. (Figura 2). 
FIGURA 2: DISTRIBUIÇAO DOS DISTRITOS E SUBDISTRITOS DO MUMICIPIO DE SAN PAULO, SEGUNDO ZONAS.

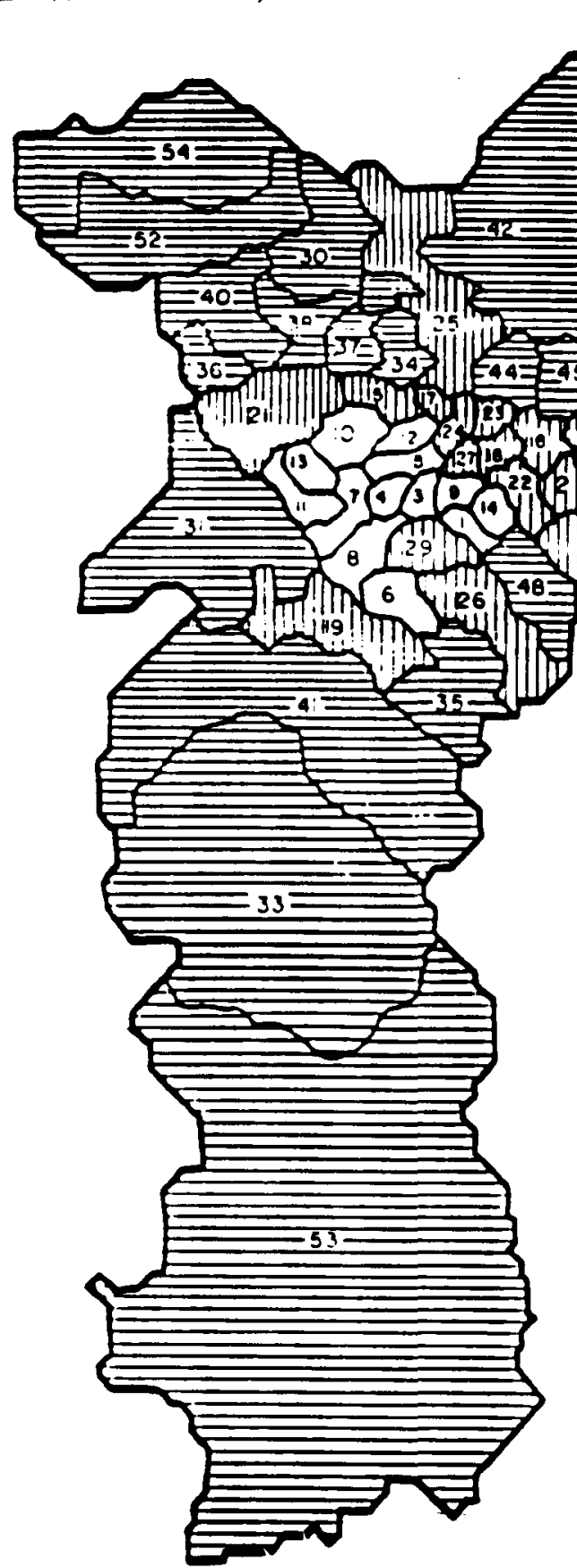

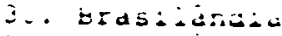

i. butanta

3. Lanisioa

j. Capela do socozzo

ji. Casa Verae

j̇. Jabaquara

30. Jaguara

37. Limāo

38. Nossa Senhora do 0

3y. Penria de França

4i. Piritudá

4.. Santo Amaro

42. Tucuruvi

43. Viá formosa

6i. Vila Guilnerme

is. Visa Marla

ic. Vila Mat:ios

:7. Vila Nova Cacnoesrinna

4c. V'ila pruaente

4y. Ermel inao Matarazzo

sc. Gualanazes

$\therefore$ I taquera

: . Jaraquá

5j. Parelne1ros

si. Perus

55. Sào Mlquel Paidista

5o. Italm kauld6ta 
O Subdistrito do Butantã colaborou com o maior número de casos, 20 (9,9\%), seguido do Subdistrito de Santo Amaro com 18 (8,9\%) e Subdistrito da Sé com 17 (8,4\%) no quinqüênio, 1985 - 1989. Nas tabelas $6 a$ e 6b ao analisarmos o ano de 1985 verificamos uma concentração no Subsdistrito da Sé com 7 casos e subdistrito do Butantã e Tucuruvi com 5 casos cada.

Em 1986 o Subdistrito da Sé apresentou 9 casos (16,7\%) seguido pelo Subdistrito de Santo Amaro com 7 (13,0\%). Com exceção dos Subdistritos do Belenzinho, de Vila Brasilândia, do Limão e Vila Formosa, n o período de 1985 a 1989, todos os outros Subdistritos produziram casos. O Distrito de São Paulo acumulou 93,1\% dos casos de Cancro Mole registrados no Município de São Paulo. Dos 11 Distritos, 4 (36,4\%) Guainazes, Jaraguá, Perus e Sapopemba, não produziram casos. O Centro histórico de São Paulo, abrangendo o Subdistrito de Sé, passou por um processo de deterioração, sendo inicialmente local de escritórios, passou a dar lugar a habitações mais baratas, tendo concentrado nos anos de $1985 \mathrm{e}$ 1986,16 casos $(94,1 \%)$ dos 17 registrados no quinqüênio.

A Secretaria de Estado da Saúde de São Paulo divide o Município de São Paulo em Escritórios Regionais de Saúde (ERSA) de 1 a 8 (Figura 3). Na Tabela 7 podemos observar uma concentração nos anos de 1985, 1986 e 1988 no ERSA-1 e 1987 e 1989 no ERSA-2. No quinqüênio, $36,6 \%$ dos casos de Cancro Mole concentraram-se no ERSA-1. Os ERSA-4 e 7 foram os que apresentaram o menor índice de casos, 8 (4,0\%), no período. Em 1987, o ERSA-6 e o ERSA-5 em 1989, não apresentaram casos. 
FIGURA 3: DISTRIBUIÇAO DOS ESCRITORIOS REGIONAIS DE SAODE DO MUNICIPIO DE SAO PAULO, POR ZONA

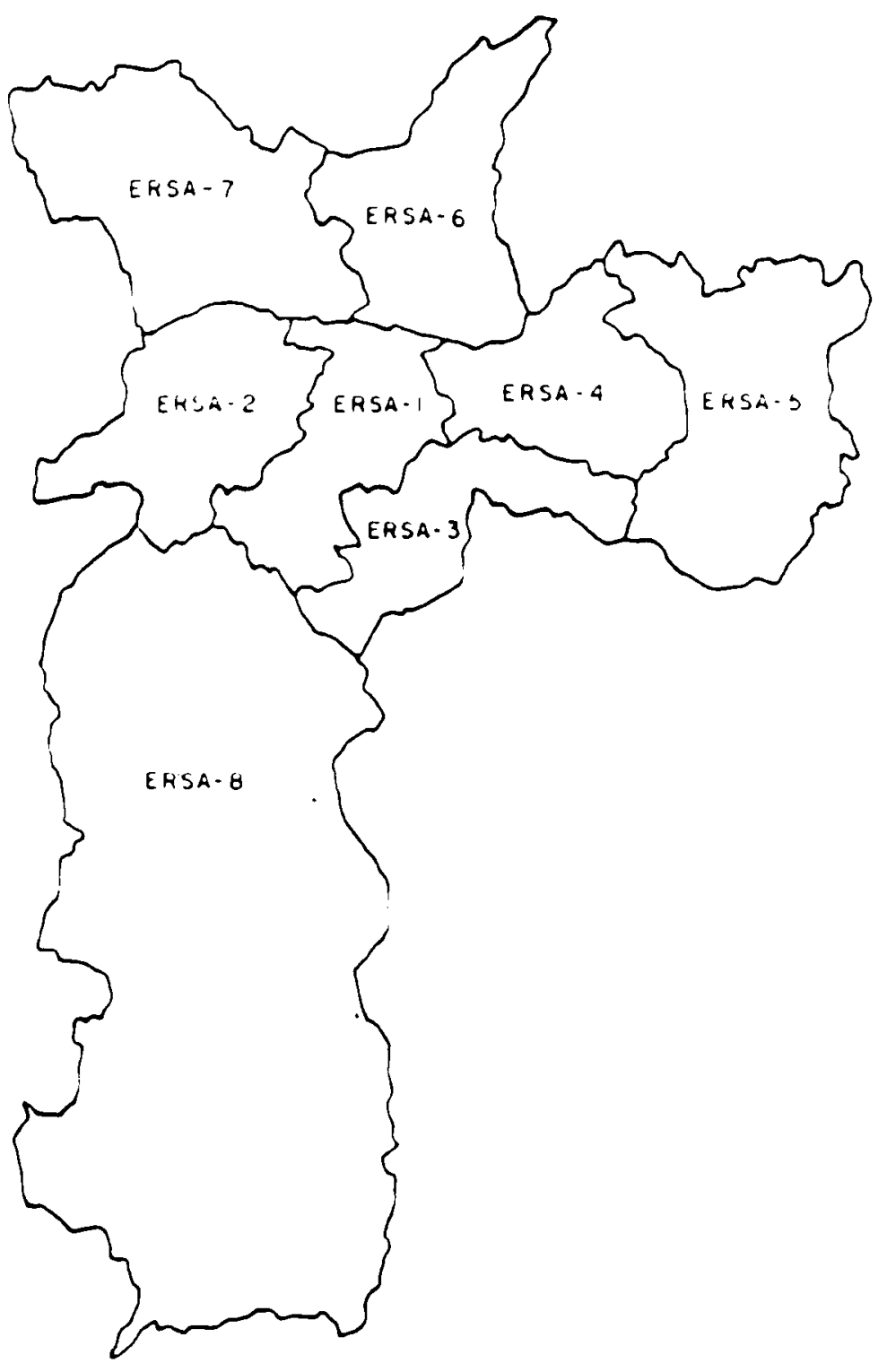

FONTE. SERVICYO DE VIGILĀNCIA EPIDEMIOLOGICA SSISP. 
Não só pela distribuição de Distritos e Subdistritos como pelos Escritórios Regionais de Saúde, o Serviço de Dermatologia Sanitária do Centro de Saúde Escola "Geraldo Horácio de Paula Souza", recebeu pacientes de quase todos os pontos do Município, esta abrangência 0 reforça como Centro de Referência.

$\mathrm{Na}$ Tabela 8 distribuiu-se os Distritos e Subdistritos pelos Escritórios Regionais de Saúde de 1 a 8.

O ERSA-1, O mais freqüente, engloba os Subdistritos da Aclimação, Barra Funda, Bela Vista, Bom Retiro, Brás, Cambuci, Cerqueira Cesar, Consolação, Ibirapuera, Indianópolis, Jardim Paulista, Liberdade, Móoca, Pari, Santa Cecília, Santa Efigênia, Sé e Vila Mariana, sendo o Pari o único Subdistrito onde não foi registrado casos. 
Tabela - 1 - Distribuição dos diagnósticos de Cancro Mole realizados no município de São Paulo - Serviço de Dermatologia Sanitária da Faculdade de Saúde Pública da Universidade de São Paulo, por faixa etária, 1985 - 1989.

\begin{tabular}{|c|c|c|c|c|c|c|c|c|c|c|c|c|}
\hline ANO & & & & & & & & & & & & \\
\hline ETARIA & № & $\%$ & $\mathrm{~N}^{\mathrm{e}}$ & $\%$ & No & $\%$ & No & $\%$ & № & $\%$ & No & $\%$ \\
\hline $15-19$ & 4 & 6,3 & 13 & 24,1 & 3 & 11,5 & 2 & 5,1 & 6 & 30,0 & 28 & 13,9 \\
\hline $20-24$ & 20 & 31,7 & 15 & 27,8 & 11 & 42,3 & 10 & 25,6 & 7 & 35,0 & 63 & 31,2 \\
\hline $25-29$ & 24 & 38,1 & 18 & 33,3 & 6 & 23,1 & 13 & 33,3 & 6 & 30,0 & 67 & 33,2 \\
\hline $30-34$ & 9 & 14,3 & 5 & 9,3 & 2 & 7,7 & 7 & 17,9 & - & $\cdots$ & 23 & 11,3 \\
\hline $35-39$ & 3 & 4,8 & 2 & 3,7 & 1 & 3,8 & 5 & 12,8 & - & $-\cdots$ & 11 & 5,4 \\
\hline $40-44$ & 1 & 1,6 & - & - & - & $\ldots$ & - & ...- & 1 & 5,0 & 2 & 1,0 \\
\hline $45-49$ & 1 & 1,6 & - & --- & 1 & 3,8 & 1 & 2,6 & - & $\cdots$ & 3 & 1,5 \\
\hline $50-54$ & 1 & 1,6 & 1 & 1,8 & 1 & 3,8 & - & -.-- & - & $-\cdots$ & 3 & 1,5 \\
\hline $55-59$ & - & $\ldots$ & - & .... & - & $\ldots$ & 1 & 2,6 & - & -..- & 1 & 0,5 \\
\hline$\geq 60$ & - & --- & - & --- & 1 & 3,8 & - & --- & - & --- & 1 & 0,5 \\
\hline TOTAL & 63 & 100,0 & 54 & 100,0 & 26 & 100,0 & 39 & 100,0 & 20 & 100,0 & 202 & 100,0 \\
\hline
\end{tabular}

Fonte: Arquivo da Dermatologia Sanitária - CSE Geraldo Paula Souza - FSP - USP 
Tabela - 2 - Distribuição dos diagnósticos de Cancro Mole realizados no município de São Paulo - Serviço de Dermatologia Sanitária da Faculdade de Saúde Pública da Universidade de São Paulo, por sexo, 1985 - 1989.

\begin{tabular}{|c|c|c|c|c|c|c|c|c|c|c|c|c|}
\hline \multirow[t]{2}{*}{ ANO } & \multicolumn{2}{|c|}{1985} & \multicolumn{2}{|c|}{1986} & \multicolumn{2}{|c|}{1987} & \multicolumn{2}{|c|}{1988} & \multicolumn{2}{|c|}{1989} & \multicolumn{2}{|c|}{ TOTAL } \\
\hline & No & $\%$ & $\mathrm{~N}^{\mathrm{e}}$ & $\%$ & $\mathrm{~N}^{\mathrm{o}}$ & $\%$ & Ne & $\%$ & No & $\%$ & № & $\%$ \\
\hline MASCULINO & 60 & 95,2 & 52 & 96,3 & 19 & 73,1 & 31 & 79,5 & 16 & 80,0 & 178 & 88,1 \\
\hline FEMININO & 3 & 4,8 & 2 & 3,7 & 7 & 26,9 & 8 & 20,5 & 4 & 20,0 & 24 & 11,9 \\
\hline TOTAL & 63 & 100,0 & 54 & 100,0 & 26 & 100,0 & 39 & 100,0 & 20 & 100,0 & 202 & 100,0 \\
\hline
\end{tabular}

Fonte: Arquivo da Dermatologia Sanitária - CSE Geraldo Paula Souza - FSP - USP 
TABELA - 3 - Distribuição dos diagnósticos de Cancro Mole realizados no Município de São Paulo - Serviço de Dermatologia Sanitária da Faculdade de Saúde Pública da Universidade de São Paulo, por raça, 1985 - 1989.

\begin{tabular}{|c|c|c|c|c|c|c|c|c|c|c|c|c|}
\hline \multirow[t]{2}{*}{ ANO } & \multicolumn{2}{|c|}{1985} & \multicolumn{2}{|c|}{1986} & \multicolumn{2}{|c|}{1987} & \multicolumn{2}{|c|}{1988} & \multicolumn{2}{|c|}{1989} & \multicolumn{2}{|c|}{ TOTAL } \\
\hline & $\mathrm{N}^{2}$ & $\%$ & № & $\%$ & No & $\%$ & $\mathrm{~N}^{\circ}$ & $\%$ & $\mathrm{~N}^{\mathrm{Q}}$ & $\%$ & № & $\%$ \\
\hline BRANCA & 43 & 68,3 & 35 & 64,8 & 18 & 69,2 & 18 & 46,1 & 9 & 45,0 & 123 & 60,9 \\
\hline NEGRA & 8 & 12,7 & 6 & 11,1 & 2 & 7,7 & 11 & 28,2 & 5 & 25,0 & 32 & 15,8 \\
\hline MULATO & 11 & 17,5 & 12 & 22,2 & 6 & 23,1 & 9 & 23,1 & 6 & 30,0 & 44 & 21,8 \\
\hline AMARELA & 1 & 1,5 & 2 & 1,9 & - & -- & 1 & 2,6 & - & $\ldots$ & 3 & 1,5 \\
\hline TOTAL & 63 & 100,0 & 54 & 100,0 & 26 & 100,0 & 39 & 100,0 & 20 & 100,0 & 202 & 100,0 \\
\hline
\end{tabular}

Fonte: Arquivo da Dermatologia Sanitária - CSE Geraldo Paula Souza - FSP - USP 
TABELA - 4 - Distribuição dos diagnósticos de Cancro Mole realizados no Municlpio de São Paulo - Serviço de Dermatologia Sanitária da Faculdade de Saúde Pública da Universidade de São Paulo, por estado civil, 1985 - 1989.

\begin{tabular}{|c|c|c|c|c|c|c|c|c|c|c|c|c|}
\hline \multirow{2}{*}{$\begin{array}{l}\text { ESTADO } \\
\text { CIVIL }\end{array}$} & \multicolumn{2}{|c|}{1985} & \multicolumn{2}{|c|}{1986} & \multicolumn{2}{|c|}{1987} & \multicolumn{2}{|c|}{1988} & \multicolumn{2}{|c|}{1989} & \multicolumn{2}{|c|}{ TOTAL } \\
\hline & № & $\%$ & Ne & $\%$ & № & $\%$ & $\mathrm{~N}^{2}$ & $\%$ & № & \% & Ne & $\%$ \\
\hline SOLTEIRO & 51 & 80,9 & 47 & 87,0 & 20 & 77,0 & 26 & 66,6 & 17 & 85,0 & 161 & 79,7 \\
\hline CASADO & 11 & 17,5 & 7 & 13,0 & 4 & 15,4 & 11 & 28,2 & 1 & 5,0 & 34 & 16,8 \\
\hline AMASIADO & - & $\cdots$ & - & ---- & -- & $-\cdots$ & 1 & 2,6 & 2 & 10,0 & 3 & 1,5 \\
\hline DESQUITADO & 1 & 1,6 & - & --- & 1 & 3,8 & 1 & 2,6 & - & $\cdots$ & 3 & 1,5 \\
\hline VIÚVO & $=$ & $\ldots$ & - & $\ldots$ & 1 & 3,8 & -- & $\ldots$ & - & $\ldots$ & 1 & 0,5 \\
\hline TOTAL & 63 & 100,0 & 54 & 100,0 & 26 & 100,0 & 39 & 100,0 & 20 & 100,0 & 202 & 100,0 \\
\hline
\end{tabular}

Fonte: Arquivo da Dermatologia Sanitária - CSE Geraldo Paula Souza - FSP - USP 
TABELA - 5 - Distribuição dos diagnósticos de Cancro Mole realizados no Município de São Paulo - Serviço de Dermatologia Sanitária da Faculdade de Saúde Pública da Universidade de São Paulo, por escolaridade, 1985 - 1989.

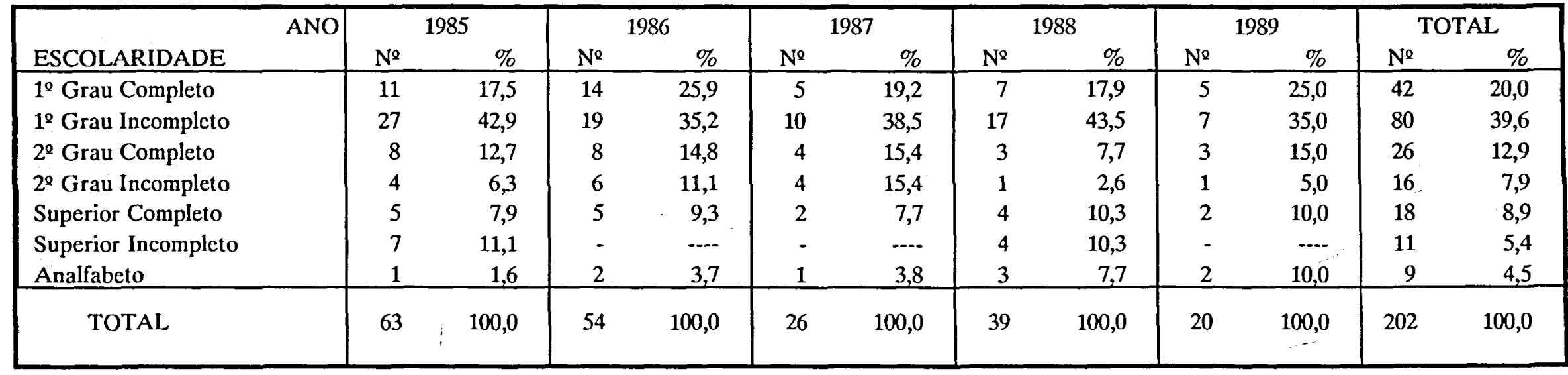

Fonte: Arquivo da Dermatologia Sanitária - CSE Geraldo Paula Souza - FSP - USP 
TABELA - 6.A - Distribuição dos diagnósticos de Cancro Mole realizados no Município de São Paulo - Serviço de Dermatologia Sanitária da Faculdade de Saúde Pública da Universidade de São Paulo, por subdistrito de residência, 1985 - 1989.

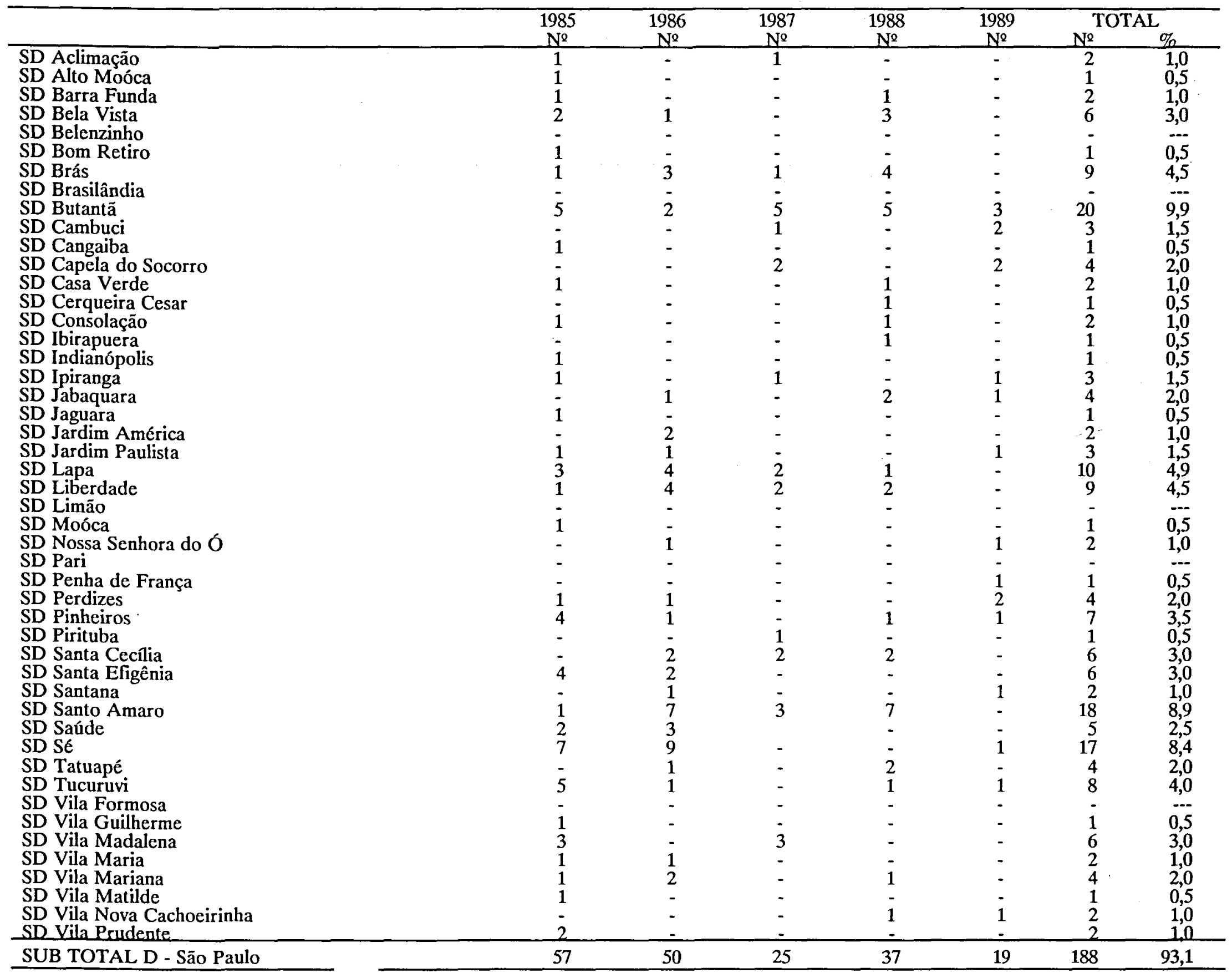


TABELA - 6.B - Distribuição dos diagnósticos de Cancro Mole realizados no Município de São Paulo - Serviço de Dermatologia Sanitária da Faculdade de Saúde Pública da Universidade de São Paulo, por distrito de residência, 1985 - 1989.

\begin{tabular}{|c|c|c|c|c|c|c|c|}
\hline & $\begin{array}{c}1985 \\
\mathrm{~N}^{\mathrm{e}}\end{array}$ & $\begin{array}{c}1986 \\
\text { № } \\
\end{array}$ & $\begin{array}{c}1987 \\
N^{0} \\
\end{array}$ & $\begin{array}{c}1988 \\
\mathrm{~N}^{\mathrm{e}} \\
\end{array}$ & $\begin{array}{c}1989 \\
\mathrm{~N}^{\mathrm{e}} \\
\end{array}$ & № & $\begin{array}{r}\text { TOTAL } \\
\% \\
\end{array}$ \\
\hline D Ermelino Matarazzo & 1 & - & - & 1 & - & 2 & 1,0 \\
\hline D Guaianazes & - & - & - & - & - & - & -- \\
\hline D Itaim Paulista & 1 & 1 & 1 & - & - & 3 & 1,5 \\
\hline D Itaquera & 1 & - & - & 1 & - & 2 & 1,0 \\
\hline D Jaraguá & - & - & - & - & - & - & $\ldots$ \\
\hline D Parelheiros & 2 & - & - & - & 1 & 3 & 1,5 \\
\hline D Perus & - & - & - & - & - & - & -- \\
\hline D São Miguel Paulista & 1 & 2 & - & - & - & 3 & 1,5 \\
\hline D Sāo Mateus & - & 1 & - & - & - & 1 & 0,5 \\
\hline D Sapopemba & - & - & - & - & - & - &.- \\
\hline SUB TOTAL & 6 & 4 & 1 & 2 & 1 & 14 & 7,0 \\
\hline TOTAL & 63 & 54 & 26 & 39 & 20 & 202 & 100,0 \\
\hline
\end{tabular}


TABELA - 7 - Distribuição dos diagnósticos de Cancro Mole realizados no Município de São Paulo - Serviço de Dermatologia Sanitária da Faculdade de Saúde Pública da Universidade de São Paulo, por Escritório Regional de Saúde (ERSA), 1985 - 1989.

\begin{tabular}{|c|c|c|c|c|c|c|c|c|c|c|c|c|}
\hline \multirow{2}{*}{$\begin{array}{l}\text { ANO } \\
\text { ESCRITÓRIO } \\
\text { REGIONAL }\end{array}$} & \multicolumn{2}{|c|}{1985} & \multicolumn{2}{|c|}{1986} & \multicolumn{2}{|c|}{1987} & \multicolumn{2}{|c|}{1988} & \multicolumn{2}{|c|}{1989} & \multicolumn{2}{|c|}{ TOTAL } \\
\hline & № & $\%$ & No & $\%$ & № & $\%$ & $\mathrm{~N}^{\mathrm{O}}$ & $\%$ & № & $\%$ & № & $\%$ \\
\hline ERSA - 1 & 23 & 36,5 & 24 & 44,4 & 7 & 26,9 & 16 & 41,0 & 4 & 20,0 & 74 & 36,6 \\
\hline ERSA - 2 & 16 & 25,4 & 10 & 18,5 & 10 & 38,5 & 7 & 18,0 & 6 & 30,0 & 49 & 24,3 \\
\hline ERSA - 3 & 5 & 7,9 & 4 & 7,4 & 1 & 3,8 & 2 & 5,1 & 2 & 10,0 & 14 & 6,9 \\
\hline ERSA - 4 & 3 & 4,8 & 1 & 1,9 & 1 & 3,8 & 2 & 5,1 & 1 & 5,0 & 8 & 4,0 \\
\hline ERSA - 5 & 4 & 6,4 & 4 & 7,4 & 1 & 3,8 & 2 & 5,1 & - & --- & 11 & 5,4 \\
\hline ERSA - 6 & 7 & 11,1 & 3 & 5,6 & - & -- & 1 & 2,6 & 2 & 10,0 & 13 & 6,4 \\
\hline ERSA - 7 & 2 & 3,1 & 1 & 1,9 & 1 & 3,8 & 2 & 5,1 & 2 & 10,0 & 8 & 4,0 \\
\hline ERSA - 8 & 3 & 4,8 & 7 & 12,9 & 5 & 19,3 & 7 & 18,0 & 3 & 15,0 & 25 & 12,4 \\
\hline TOTAL & 63 & 100,0 & 54 & 100,0 & 26 & 100,0 & 39 & 100,0 & 20 & 100,0 & 202 & 100,0 \\
\hline
\end{tabular}

Fonte: Arquivo da Dermatologia Sanitária - CSE Geraldo Paula Souza - FSP - USP 
TABELA - 8 - Distribuição dos Distritos e Subdistritos do Municipio de São Paulo, Escritórios Regionais de Saúde (ERSA).

\begin{tabular}{|c|c|c|c|}
\hline \multirow[t]{3}{*}{ ERSA-1 - } & $\begin{array}{l}\text { Aclimação } \\
\text { Barra Funda } \\
\text { Bela Vista } \\
\text { Bom Retiro } \\
\text { Brás } \\
\text { Cambucí } \\
\text { Cerqueira Cesar } \\
\text { Consolação }\end{array}$ & ERSA-4 - & $\begin{array}{l}\text { Alto da Moóca } \\
\text { Belenzinho } \\
\text { Cangaíba } \\
\text { Penha de França } \\
\text { Tatuapé } \\
\text { Vila Formosa } \\
\text { Vila Matilde }\end{array}$ \\
\hline & $\begin{array}{l}\text { Ibirapuera } \\
\text { Indianópolis } \\
\text { Jardim Paulista } \\
\text { Liberdade } \\
\text { Moóca } \\
\text { Pari }\end{array}$ & ERSA-5 - & $\begin{array}{l}\text { Ermelino Matarazzo } \\
\text { Guaianazes } \\
\text { Itaim Paulista } \\
\text { Itaquera } \\
\text { São Miguel Paulista }\end{array}$ \\
\hline & $\begin{array}{l}\text { Santa Cecilia } \\
\text { Santa Efigênia } \\
\text { Sé } \\
\text { Vila Mariana }\end{array}$ & ERSA-6 - & $\begin{array}{l}\text { Santana } \\
\text { Tucuruvi } \\
\text { Vila Guilherme } \\
\text { Vila Maria }\end{array}$ \\
\hline ERSA-2 - & $\begin{array}{l}\text { Butantã } \\
\text { Jardim america } \\
\text { Lapa } \\
\text { Perdizes } \\
\text { Pinheiros } \\
\text { Vila Madalena }\end{array}$ & ERSA-7 - & $\begin{array}{l}\text { Brasilândia } \\
\text { Casa Verde } \\
\text { Jaraguá } \\
\text { Limão } \\
\text { Nossa Senhora do ó } \\
\text { Perus } \\
\text { Pirituba } \\
\text { Vila Jaguará } \\
\text { Vila Nova Cachoeirinha }\end{array}$ \\
\hline -3- & $\begin{array}{l}\text { lpiranga } \\
\text { Jabaquara } \\
\text { Saúde } \\
\text { Vila Prudente }\end{array}$ & ERSA-8 - & $\begin{array}{l}\text { Parelheiros } \\
\text { Santo Amaro } \\
\text { Capela do Socorro }\end{array}$ \\
\hline
\end{tabular}

Fonte: Centro de Vigilância Sanitária. 
Na Tabela 9 observa-se que o hábito sexual predominante foi o heterossexual (86,6\%) com uma variação de 76,9\% em 1987 e 93,7\% em 1985. Referiam homossexualidade masculina exclusiva 16 (7,9\%) e 5,5\% a bissexualidade. Dos 202 casos observados apenas 1 apresentou lesão anal.

Um dos fatores que favorece o recrudescimento das DST é o Início Sexual cada vez mais precoce. $\mathrm{Na}$ Tabela 10 podemos observar $31,6 \%$ do total de pacientes portadores de Cancro Mole tiveram o início sexual com menos de 15 anos, com um destaque para o ano de 1987 onde $65,4 \%$ teve o início entre 9 e 14 anos, contrastando com 4,5\% que tiveram o seu primeiro contato entre 20 e 26 anos. A idade mais freqüente no quinqüênio foi 14 anos, acompanhada em 1988 de 12 anos em 1985 e 1989 de 16 anos.

A Tabela 11 demonstra que $79(39,1 \%)$ dos pacientes não referiam passado venéreo, o que indica que o episódio detectado de Cancro Mole é o primeiro acidente venéreo, havendo uma variação crescente de $27,8 \%$ em 1985 e $60 \%$ em 1989. Em 34\% referiam 1 episódio e em $26,2 \%$ mais de 1 episódio.

Com relação ao primeiro episódio venéreo, na Tabela 12 a gonorréia foi a mais freqüente em todos os anos com 64 episódios e o Cancro Mole foi referido em $24(19,7 \%)$ dos pacientes, o que leva a supor sobre possiveis recidivas. Apesar da diminuição do número de casos registrados de gonorréia na $2^{\mathrm{a}}$ metade da década de 80 , verifica-se que a gonorréia continua sendo a mais freqüente.

Acreditamos que os parâmetros sociais acabam por determinar a origem dos surtos, provavelmente aspecto que contribui é a promiscuidade sexual caracterizada por elevado número de DST pregressas encontradas 
neste grupo. Podemos observar a distribuição da faixa etária do primeiro episódio venéreo na Tabela 13, onde verificamos concentração no período dos 15 aos 24 anos $(79,6 \%)$. 
TABELA - 9 - Distribuição dos diagnósticos de Cancro Mole realizados no Munić́pio de São Paulo - Serviço de Dermatologia Sanitária da Faculdade de Saúde Pública da Universidade de São Paulo, por hábito sexual, 1985 - 1989.

\begin{tabular}{|c|c|c|c|c|c|c|c|c|c|c|c|c|}
\hline \multirow{2}{*}{$\begin{array}{ll} & \text { ANO } \\
\text { HÁBITO } & \\
\text { SEXUAL } & \\
\end{array}$} & \multicolumn{2}{|c|}{1985} & \multicolumn{2}{|c|}{1986} & \multicolumn{2}{|c|}{1987} & \multicolumn{2}{|c|}{1988} & \multicolumn{2}{|c|}{1989} & \multicolumn{2}{|c|}{ TOTAL } \\
\hline & № & $\%$ & № & $\%$ & № & $\%$ & No & $\%$ & № & $\%$ & № & $\%$ \\
\hline Heterossexual & 59 & 93,7 & 46 & 85,2 & 20 & 76,9 & 32 & 82,0 & 18 & 90,0 & 175 & 86,6 \\
\hline Homossexual & 1 & 1,6 & 3 & 5,6 & 4 & 15,4 & 7 & 18,0 & 1 & 5,0 & 16 & 7,9 \\
\hline Bissexual & 3 & 4,7 & 5 & 9,2 & 2 & 7,7 & $=$ & $\cdots$ & 1 & 5,0 & 11 & 5,5 \\
\hline TOTAL & 63 & 100,0 & 54 & 100,0 & 26 & 100,0 & 39 & 100,0 & 20 & 100,0 & 202 & 100,0 \\
\hline
\end{tabular}

Fonte: Arquivo da Dermatologia Sanitária - CSE Geraldo Paula Souza - FSP - USP 
TABELA - 10 - Distribuição dos diagnósticos de Cancro Mole realizados no Município de São Paulo - Serviço de Dermatologia Sanitária da Faculdade de Saúde Pública da Universidade de São Paulo, por idade do início sexual, 1985 - 1989.

\begin{tabular}{|c|c|c|c|c|c|c|c|c|c|c|c|c|}
\hline \multirow{2}{*}{$\begin{array}{ll}\text { IDADE } & \text { ANO } \\
\text { (ANOS) } & \\
\end{array}$} & \multicolumn{2}{|c|}{1985} & \multicolumn{2}{|c|}{1986} & \multicolumn{2}{|c|}{1987} & \multicolumn{2}{|c|}{1988} & \multicolumn{2}{|c|}{1989} & \multicolumn{2}{|c|}{ TOTAL } \\
\hline & № & $\%$ & № & $\%$ & № & $\%$ & № & $\%$ & No & $\%$ & № & $\%$ \\
\hline 9 & 1 & 1,6 & 2 & 3,7 & 1 & 3,8 & 1 & 2,6 & - & --- & 5 & 2,5 \\
\hline 10 & - & --- & - & $\ldots$ & 1 & 3,8 & - & --- & - & $\cdots$ & 1 & 0,5 \\
\hline 11 & 2 & 3,2 & - & -.. & - & -- & - & $\cdots$ & 1 & 5,0 & 3 & 1,5 \\
\hline 12 & 1 & 1,6 & 2 & 3,7 & 4 & 15,5 & 6 & 15,3 & 3 & 15,0 & 16 & 7,9 \\
\hline 13 & 6 & 9,5 & 10 & 18,5 & 2 & 7,7 & 1 & 2,6 & 2 & 10,0 & 21 & 10,4 \\
\hline 14 & 8 & 12,7 & 11 & 20,4 & 9 & 34,6 & 6 & 15,3 & 4 & 20,0 & 38 & 18,8 \\
\hline 15 & 12 & 19,0 & 8 & 14,8 & 1 & 3,8 & 5 & 12,8 & 3 & 15,0 & 29 & 14,3 \\
\hline 16 & 13 & $20,6 !$ & 6 & 11,1 & 1 & 3,8 & 3 & 7,7 & 4 & 20,0 & 27 & 13,4 \\
\hline 17 & 8 & 12,7 & 10 & 18,5 & 4 & 15,5 & 5 & 12,8 & 1 & 5,0 & 28 & 13,8 \\
\hline 18 & 8 & 12,7 & 3 & 5,5 & 2 & 7,7 & 6 & 15,3 & 1 & 5,0 & 20 & 9,9 \\
\hline 19 & 2 & 3,2 & 1 & 1,9 & - & --- & 2 & 5,1 & - & --- & 5 & 2,5 \\
\hline 20 & 1 & 1,6 & - & -- & 1 & 3,8 & 1 & 2,6 & 1 & 5,0 & 4 & 2,0 \\
\hline 21 & 1 & 1,6 & $-\cdot$ & $\cdots$ & - & $\cdots$ & - & --- & - & --. & 1 & 0,5 \\
\hline 22 & - & --- & 1 & 1,9 & - & --- & 1 & 2,6 & - & --- & 2 & 1,0 \\
\hline 24 & - & --- & - & --- & - & --- & 1 & 2,6 & - & --- & 1 & 0,5 \\
\hline 26 & - & $\ldots$ & - & $\cdots$ & - & $\cdots$ & 1 & 2,6 & - & --- & 1 & 0,5 \\
\hline TOTAL & 63 & 100,0 & 54 & 100,0 & 26 & 100,0 & 39 & 100,0 & 20 & 100,0 & 202 & 100,0 \\
\hline
\end{tabular}

Fonte: Arquivo da Dermatologia Sanitária - CSE Geraldo Paula Souza - FSP - USP 
TABELA - 11 - Distribuição dos diagnósticos de Cancro Mole realizados no Município de São Paulo - Serviço de Dermatologia Sanitária da Faculdade de Saúde Pública da Universidade de São Paulo, por número, pregresso de episódio venéreos, 1985 - 1989.

\begin{tabular}{|c|c|c|c|c|c|c|c|c|c|c|c|c|}
\hline \multirow{2}{*}{$\begin{array}{ll} & \text { ANO } \\
\text { NÚMEROS } & \\
\text { EPISODIOS } & \\
\end{array}$} & \multicolumn{2}{|c|}{1985} & \multicolumn{2}{|c|}{1986} & \multicolumn{2}{|c|}{1987} & \multicolumn{2}{|c|}{1988} & \multicolumn{2}{|c|}{1989} & \multicolumn{2}{|c|}{ TOTAL } \\
\hline & № & $\%$ & Ne & $\%$ & Ne & $\%$ & Ne & $\%$ & Ne & $\%$ & № & $\%$ \\
\hline 0 & 25 & 39,7 & 15 & 27,8 & 9 & 34,7 & 18 & 46,2 & 12 & 60,0 & 79 & 39,1 \\
\hline 1 & 30 & 47,6 & 24 & 44,4 & 10 & 38,5 & 6 & 15,4 & - & -.. & 70 & 34,7 \\
\hline 2 & 4 & 6,3 & 12 & 22,2 & 5 & 19,2 & 9 & 23,0 & 3 & 15,0 & 33 & 16,3 \\
\hline 3 & 1 & 1,6 & 3 & 5,6 & 1 & 3,8 & 2 & 5,1 & 5 & 25,0 & 12 & 5,9 \\
\hline 4 & 1 & 1,6 & - & $\cdots$ & 1 & 3,8 & 2 & 5,1 & - & $\ldots$ & 4 & 2,0 \\
\hline 5 & - & $\ldots$ & - & -.- & - & $\ldots$ & 1 & 2,6 & - & $\cdots$ & 1 & 0,5 \\
\hline+5 & 2 & 3,2 & - & $-\ldots$ & - & $\ldots$ & 1 & 2,6 & $=$ & $\ldots$ & 3 & 1,5 \\
\hline TOTAL & 63 & 100,0 & 54 & 100,0 & 26 & 100,0 & 39 & 100,0 & 20 & 100,0 & 202 & 100,0 \\
\hline
\end{tabular}

Fonte: Arquivo da Dermatologia Sanitária - CSE Geraldo Paula Souza - FSP - USP 
TABELA - 12 - Distribuição dos diagnósticos de Cancro Mole realizados no Município de São Paulo - Serviço de Dermatologia Sanitária da Faculdade de Saúde Pública da Universidade de São Paulo, por diagnóstico do primeiro episódio venéreo, 1985 - 1989.

\begin{tabular}{|c|c|c|c|c|c|c|c|c|c|c|c|c|}
\hline \multirow{2}{*}{ DIAGNÓSTICO } & \multicolumn{2}{|c|}{1985} & \multicolumn{2}{|c|}{1986} & \multicolumn{2}{|c|}{1987} & \multicolumn{2}{|c|}{1988} & \multicolumn{2}{|c|}{1989} & \multicolumn{2}{|c|}{ TOTAL } \\
\hline & № & $\%$ & No & $\%$ & № & $\%$ & No & $\%$ & $\mathrm{~N}^{\mathrm{Q}}$ & $\%$ & № & $\%$ \\
\hline Gonorréia & 23 & 60,5 & 17 & 44,7 & 8 & 47,1 & 9 & 42,8 & 7 & 87,5 & 64 & 52,5 \\
\hline Sifflis & 6 & 15,8 & 5 & 13,2 & 1 & 5,9 & 5 & 23,8 & - & $\cdots$ & 17 & 13,9 \\
\hline Cancro Mole & 6 & 15,8 & 12 & 31,6 & 4 & 23,5 & 2 & 9,5 & - & $\cdots$ & 24 & 19,7 \\
\hline Condiloma Acuminado & 2 & 5,3 & 2 & 5,3 & 3 & 17,6 & 2 & 9,5 & - & -- & 9 & 7,4 \\
\hline U.N.G. & 1 & 2,6 & - & -.. & 1 & 5,9 & 1 & 4,8 & 1 & 12,5 & 4 & 3,3 \\
\hline Herpes Genital & - & -.. & 1 & 2,6 & - & $\ldots$ & 1 & 4,8 & - & -- & 2 & 1,6 \\
\hline Linfogranuloma & - & --- & 1 & 2,6 & - & $\ldots$ & 1 & 4,8 & 二 & $\ldots$ & 2 & 1,6 \\
\hline TOTAL & 38 & 100,0 & 38 & 100,0 & 17 & 100,0 & 21 & 100,0 & 8 & 100,0 & 122 & 100,0 \\
\hline
\end{tabular}

Fonte: Arquivo da Dermatologia Sanitária - CSE Geraldo Paula Souza - FSP - USP 
TABELA - 13 - Distribuição dos diagnósticos de Cancro Mole realizados no Município de São Paulo - Serviço de Dermatologia Sanitária da Faculdade de Saúde Pública da Universidade de São Paulo, por faixa etária do primeiro episódio venéreo, 1985 - 1989.

\begin{tabular}{|c|c|c|c|c|c|c|c|c|c|c|c|c|}
\hline ANO & & & & & & & & & & & & \\
\hline ETÁRIA & No & $\%$ & Ne & $\%$ & Ne & $\%$ & Ne & $\%$ & Ne & $\%$ & № & $\%$ \\
\hline $10-14$ & - & $\cdots$ & 1 & 2,6 & - & $\ldots$ & - & $\cdots$ & 1 & 12,5 & 2 & 1,6 \\
\hline $15 \cdot 19$ & 14 & 36,8 & 22 & 57,9 & 8 & 47,0 & 8 & 38,1 & 2 & 25,0 & 54 & 44,3 \\
\hline $20-24$ & 16 & 42,1 & 11 & 28,9 & 7 & 41,2 & 6 & 28,5 & 3 & 37,5 & 43 & 35,3 \\
\hline $25-29$ & 6 & 15,8 & 3 & 7,9 & 1 & 5,9 & 5 & 23,8 & 2 & 25,0 & 17 & 13,9 \\
\hline $30-34$ & 2 & 5,3 & 1 & 2,6 & 1 & 5,9 & - & -..- & - & .... & 4 & 3,3 \\
\hline $35-39$ & - & -.- & - & $\ldots$. & - & $\ldots$ & 1 & 4,8 & - & --- & 1 & 0,8 \\
\hline $40-44$ & - & $\ldots$ & - & $-\cdots$ & - & $\ldots$ & 1 & 4,8 & - & $\ldots$ & 1 & 0,8 \\
\hline TOTAL & 38 & 100,0 & 38 & 100,0 & 17 & 100,0 & 21 & 100,0 & 8 & 100,0 & 112 & 100,0 \\
\hline
\end{tabular}

Fonte: Arquivo da Dermatologia Sanitária - CSE Geraldo Paula Souza - FSP - USP 
Desde as primeiras observações, o Cancro Mole esteve ligado à prostituição. ASIN (1952) refere uma alta prevalência em prostitutas coreanas. Em $61,3 \%$ dos casos registrados por MOREL et al (1982) no período de 1973 a 1979, tinham como provável contaminação a prostituição. Em um trabalho subsidiado pelas Forças Armadas Americanas, 0 Departamento de Saúde da Marinha detectou em 8066 mulheres, 702 (6,7\%) casos de Cancro Mole e todas eram prostitutas (LAO \& TRUSSEL, 1947). Dos 300 registros feitos em Nairobi-Kenia, 57,0\% adquiriu Cancro Mole de prostitutas (PLUMMER et al, 1983), reforçado por D'COSTA, (1985).

Em Singapura, KHOO et al (1977), pesquisando Doenças Sexualmente Trasmissiveis em 200 prostituras encontrou alta percentagem de Cancro Mole. STRAKOSCH et al (1945) e SIMONS (1947) reforçam que a prostituta é o maior reservatório de Cancro Mole.

A Tabela 14 revela que a amiga foi a fonte mais freqüente, em $35,2 \%$ dos casos, estabeleceu-se que amiga é a parceira sexual eventual sem remuneração. A prostituta foi a fonte em $31,8 \% \mathrm{em} \mathrm{1985,35,2 \%} \mathrm{em}$ 1986, $11,5 \%$ em 1987, 20,5\% em 1988 e 10,0\% em 1989, caracterizando a diminuição do seu papel como fonte principal clássica. Ressalta-se que os dados obtidos não coincidem com os encontrados em outros locais. Dos 24 casos femininos adquiriram a doença do marido $19(79,2 \%)$ e $6(20,8 \%)$ de parceiros esporádicos. Observa-se portanto a ampliação da promiscuidade, fator importante na disseminação das D S T, como um todo e em especial do Cancro Mole.

Uma das características do Cancro Mole é possuir um período provável de incubação inferior ao da Sifílis. A Tabela 15 revela uma concentração período de incubação nos primeiros 7 dias em $65,1 \%, 68,5 \%$, $84,6 \%, 69,2 \%$ e $70,0 \%$ respectivamente de 1985 a 1989 e uma média de 
$72,3 \%$. O segundo e o quinto dia foram os mais freqüentes. Estes dados são concordantes com BELDA et al (1984), BELDA JUNIOR (1985), TEIXEIRA \& ALMEIDA (1985), PASSOS et al (1987) em observações brasileiras e com MEZZADRA et al (1977), HAFIZ et al (1981), PLUMMER (1985), PIOT \& MEHEUS (1986), PLATTEAU et al (1987), FELMAN (1989).

A medida que a população recebe maior conhecimento sobre Doenças Sexualmente Transmissíveis através de Educação Sanitária, a procura ao serviço tende a ser mais rápida. O tempo de doença é apresentado na Tabela 16 e revela que $60,9 \%$ dos pacientes procuraram 0 Serviço entre 10 e 19 dias de doença e em 1989, 85,0\% procurou atendimento nos primeiros 9 dias. Verifica-se que com a diminuição observada do tempo de doença, o paciente torna-se não infectante mais rápido evitando novas contaminações.

Clinicamente o Cancro Mole é caracterizado por seu caráter autoinoculante, parâmetro que colabora no diagnóstico diferencial da Sífilis primária.

O número de lesões observadas no momento do diagnóstico, variou de 1 a 10 ulcerações. A Tabela 17 revelou que 68 (33,7\%) apresentaram lesão única. A observação anual no período, demonstra uma diminuição gradativa do percentual de lesões isoladas, apesar de $85 \%$ terem procurado o serviço na primeira semana após o aparecimento da lesão.

Estes dados são concordantes com TAYLOR et al (1984), onde observou em homens 34,0\% com úlcera única e $9 \%$ com mais de 4 lesões. STURN et al (1987) obteve $55,6 \%$ de lesões únicas, LATIF et al (1984), SEKKAT et al (1984) em Marrocos, NSANZE et al (1981) no Kenia, obtiveram $55 \%$. 
O enfartamento ingüinal, caracterizado por sinais inflamatórios de dôr, rubor e calor é conhecido como bubão. No diagnóstico diferencial esbarra-se com o linfogranuloma venéreo que tem $\circ$ bubão como característica, mas não apresenta lesão ulcerada genital e com a donovanose, que apesar das lesões serem mais extensas, não desenvolve enfartamento ingüinal.

Na Tabela 18 verificamos que 99 (49,0\%) apresentou bubão, nos casos femininos não foi observado. Em $32(15,8 \%)$ foi unilateral com predominância do direito. Esta manifestação clínica é que faz com que o paciente procure o Serviço, porque a dor incomoda, dificultando a deambulação. Não houve associação entre o número de lesões e a presença do bubão. Em alguns casos, o bubão clássico era acompanhado de uma única lesão. Estes dados são concordantes com BOPP et al (1973) que encontrou $62 \%$ de bubões no Rio Grande do Sul e com NSANZE et al (1981) no Kenia, que em 97 pacientes encontrou 47\% de adenopatia ingüinal sendo $75 \%$ unilateral. 
TABELA - 14 - Distribuição dos diagnósticos de Cancro Mole realizados no Município de São Paulo - Serviço de Dermatologia Sanitária da Faculdade de Saúde Pública da Universidade de São Paulo, por tipo fonte, 1985 - 1989.

\begin{tabular}{|c|c|c|c|c|c|c|c|c|c|c|c|c|}
\hline \multirow[b]{2}{*}{ FONTE } & \multicolumn{2}{|c|}{1985} & \multicolumn{2}{|c|}{1986} & \multicolumn{2}{|c|}{1987} & \multicolumn{2}{|c|}{1988} & \multicolumn{2}{|c|}{1989} & \multicolumn{2}{|c|}{ TOTAL } \\
\hline & № & $\%$ & № & $\%$ & № & $\%$ & Ne & $\%$ & № & $\%$ & № & $\%$ \\
\hline Prostituta & 20 & 31,8 & 19 & 35,2 & 3 & 11,5 & 8 & 20,5 & 2 & 10 & 52 & 25,7 \\
\hline Amiga & 15 & 23,8 & 18 & 33,3 & 12 & 46,2 & 15 & 38,5 & 11 & 55,0 & 71 & 35,2 \\
\hline Namorada & 12 & 19,0 & 13 & 24,1 & 10 & 38,5 & 13 & 33,3 & 6 & 30,0 & 54 & 26,7 \\
\hline Esposa & 7 & 11,1 & - &.-- & 1 & 3,8 & 1 & 2,6 & - & $\cdots$ & 9 & 4,5 \\
\hline Parceira Fixa & 9 & 14,3 & 4 & 7,4 & - &.- & 2 & 5,1 & 1 & 5,0 & 16 & 7,9 \\
\hline TOTAL & 63 & 100,0 & 54 & 100,0 & 26 & 100,0 & 39 & 100,0 & 20 & 100,0 & 202 & 100,0 \\
\hline
\end{tabular}

Fonte: Arquivo da Dermatologia Sanitária - CSE Geraldo Paula Souza - FSP - USP 
TABELA - 15 - Distribuição dos diagnósticos de Cancro Mole realizados no Município de São Paulo - Serviço de Dermatologia Sanitária da Faculdade de Saúde Pública da Universidade de São Paulo, por período de incubação, 1985 - 1989.

\begin{tabular}{|c|c|c|c|c|c|c|c|c|c|c|c|c|c|}
\hline \multirow{2}{*}{\multicolumn{2}{|c|}{ DIAS $\quad$ ANO }} & \multicolumn{2}{|c|}{1985} & \multicolumn{2}{|c|}{1986} & \multicolumn{2}{|c|}{1987} & \multicolumn{2}{|c|}{1988} & \multicolumn{2}{|c|}{1989} & \multicolumn{2}{|c|}{ TOTAL } \\
\hline & & № & $\%$ & № & $\%$ & № & $\mathscr{Z}$ & № & \% & NN & $\%$ & Ne & $\%$ \\
\hline 1 & & 2 & 3,2 & 3 & 5,6 & 3 & 11,5 & 1 & 2,6 & 2 & 10,0 & 11 & 5,4 \\
\hline 2 & & 7 & 11,1 & 13 & 24,1 & 2 & 7,7 & 5 & 12,8 & 4 & 20,0 & 31 & 15,3 \\
\hline 3 & & 7 & 11,1 & 7 & 13,0 & 3 & 11,5 & 7 & 17,9 & 2 & 10,0 & 26 & 12,8 \\
\hline 4 & & 8 & 12,7 & 3 & 5,6 & 3 & 11,5 & 4 & 10,2 & 1 & 5,0 & 19 & 9,4 \\
\hline 5 & & 6 & 9,5 & 5 & 9,3 & 9 & 34,7 & 10 & 25,6 & 1 & 5,0 & 31 & 15,3 \\
\hline 6 & & 6 & 9,5 & 2 & 3,7 & 2 & 7,7 & - & $\cdots$ & 2 & 10,0 & 12 & 5,9 \\
\hline 7 & & 5 & 7,9 & 4 & 7,4 & - & $-\infty$ & - & ---- & 2 & 10,0 & 11 & 54 \\
\hline 8 & & 3 & 4,8 & 1 & 1,8 & - & -..- & - & --- & 1 & 5,0 & 5 & 2,5 \\
\hline 9 & & - & $\cdots$ & 2 & 3,7 & 1 & 3,8 & - & $\cdots$ & - & $\cdots$ & 3 & 1,5 \\
\hline 10 & & 3 & 4,8 & 4 & 7,4 & - & $\ldots$ & 3 & 7,7 & - & $-\ldots$ & 10 & 5,0 \\
\hline 11 & & - & $\cdots$ & - & $\cdots$ & - & $-\cdots$ & - & $\cdots$ & - & $\cdots-$ & - & $\cdots$ \\
\hline 12 & & 2 & 3,2 & 1 & 1,8 & - & $\cdots$ & - & $\cdots$ & - & $\cdots$ & 3 & 1,5 \\
\hline 13 & & 1 & 1,6 & - & --- & - & $\ldots$ & - & -..- & - & $\ldots$ & 1 & 0,5 \\
\hline 14 & & 3 & 4,8 & - & $\cdots$ & - & $\ldots$ & - & $\cdots$ & - & $\cdots$ & 3 & 1,5 \\
\hline 15 & & 2 & 3,2 & 1 & 1,8 & 1 & 3,8 & 3 & 7,7 & 2 & 10,0 & 9 & 4,5 \\
\hline 16 & & - & $\ldots$ & - & $\cdots$ & 1 & 3,8 & 3 & 7,7 & - & $\cdots$ & 4 & 2,0 \\
\hline 17 & & - & --- & 2 & 3,7 & - & --- & 1 & 2,6 & - & $\cdots$ & 3 & 1,5 \\
\hline 18 & & - & -.-- & 1 & 1,8 & - & $-\cdots$ & - & --. & 1 & 5,0 & 2 & 1,0 \\
\hline 19 & & 1 & 1,6 & - & -..- & - & -..- & - & --- & - & $-\cdots$ & 1 & 0,5 \\
\hline 20 & & 2 & 3,2 & - & $\cdots$ & - & $\cdots$ & - & -..- & - & $\cdots$ & 2 & 1,0 \\
\hline 21 & & 2 & 3,2 & - & -..- & - & $\ldots$ & - & $\cdots$ & - & $\cdots$ & 2 & 1,0 \\
\hline 22 & & - & -- & 2 & 3,7 & - & ---- & - & $\cdots$ & - & --. & 2 & 1,0 \\
\hline 24 & & - & -.- & 1 & 1,8 & - & $\ldots$ & - & --. & - & --- & 1 & 0,5 \\
\hline 25 & & - & --.- & 2 & 3,7 & - & -..- & . & $\cdots$ & - & $\cdots$ & 2 & 1,0 \\
\hline 26 & & 1 & 1,6 & - & $\cdots$ & - & $\cdots$ & 1 & 2,6 & - & $\cdots$ & 2 & 1,0 \\
\hline 30 & & 1 & 1,6 & - & --.- & 1 & 3,8 & 1 & 2,6 & 2 & 10,0 & 5 & 2,5 \\
\hline 41 & & 1 & 1,6 & . & $\ldots$ & & $\ldots$ & 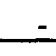 & $\ldots$ & - & $\ldots$ & 1 & 0,5 \\
\hline TOTAL & & 63 & 100,0 & 54 & 100,0 & 26 & 100,0 & 39 & 100,0 & 20 & 100,0 & 202 & 100,0 \\
\hline
\end{tabular}

Fonte: Arquivo da Dermatologia Sanitária - CSE Geraldo Paula Souza - FSP - USP 
TABELA - 16 - Distribuição dos diagnósticos de Cancro Mole realizados no Município de São Paulo - Serviço de Dermatologia Sanitária da Faculdade de Saúde Pública da Universidade de São Paulo, por tempo de doença, 1985 - 1989.

\begin{tabular}{|c|c|c|c|c|c|c|c|c|c|c|c|c|}
\hline \multirow[t]{2}{*}{ ANO } & \multicolumn{2}{|c|}{1985} & \multicolumn{2}{|c|}{1986} & \multicolumn{2}{|c|}{1987} & \multicolumn{2}{|c|}{1988} & \multicolumn{2}{|c|}{1989} & \multicolumn{2}{|c|}{ TOTAL } \\
\hline & Ne & $\%$ & $\mathrm{~N}^{2}$ & $\%$ & № & $\%$ & № & $\%$ & $\mathrm{~N}^{\mathrm{Q}}$ & $\%$ & Ne & $\%$ \\
\hline $1-14$ & 2 & 3,2 & 5 & 9,3 & 3 & 11,5 & 9 & 23,1 & 7 & 35,0 & 26 & 12,9 \\
\hline $5-9$ & 4 & 6,3 & 6 & 11,1 & 5 & 19,2 & 7 & 17,9 & 10 & 50,0 & 32 & 15,8 \\
\hline $10-14$ & 17 & 27,0 & 15 & 27,8 & 10 & 38,5 & 12 & 30,8 & 2 & 10,0 & 56 & 27,7 \\
\hline $15-19$ & 30 & 47,6 & 20 & 37,0 & 6 & 23,1 & 10 & 25,6 & 1 & 5,0 & 67 & 33,2 \\
\hline$\geq 20$ & 10 & 15,9 & 8 & 14,8 & 2 & 7,7 & 1 & 2,6 & - & $\ldots$ & 21 & 10,4 \\
\hline TOTAL & 63 & 100,0 & 54 & 100,0 & 26 & 100,0 & 39 & 100,0 & 20 & 100,0 & 202 & 100,0 \\
\hline
\end{tabular}

Fonte: Arquivo da Dermatologia Sanitária - CSE Geraldo Paula Souza - FSP - USP 
TABELA - 17 - Distribuição dos diagnósticos de Cancro Mole realizados no Munićpio de São Paulo - Serviço de Dermatologia Sanitária da Faculdade de Saúde Pública da Universidade de São Paulo, por número de lesões, 1985 - 1989.

\begin{tabular}{|c|c|c|c|c|c|c|c|c|c|c|c|c|}
\hline \multirow{2}{*}{ № de LESŌES $^{\text {ANO }}$} & \multicolumn{2}{|c|}{1985} & \multicolumn{2}{|c|}{1986} & \multicolumn{2}{|c|}{1987} & \multicolumn{2}{|c|}{1988} & \multicolumn{2}{|c|}{1989} & \multicolumn{2}{|c|}{ TOTAL } \\
\hline & № & $\%$ & $N^{2}$ & $\%$ & № & $\%$ & № & $\%$ & № & $\%$ & № & $\%$ \\
\hline 1 & 30 & 47,6 & 14 & 25,9 & 10 & 38,5 & 11 & 28,2 & 3 & 15,0 & 68 & 33,7 \\
\hline 2 & 8 & 11,2 & 14 & 25,9 & 5 & 19,2 & 5 & 12,8 & 3 & 15,0 & 34 & 16,8 \\
\hline 3 & 6 & 9,5 & 6 & 11,1 & 3 & 11,6 & 7 & 18,0 & 6 & 30,0 & 28 & 13,9 \\
\hline 4 & 6 & 9,5 & 8 & 14,8 & 1 & 3,8 & 2 & 5,1 & 1 & 5,0 & 18 & 8,9 \\
\hline 5 & 5 & 7,9 & 3 & 5,5 & - & --- & 4 & 10,3 & 2 & 10,0 & 14 & 6,9 \\
\hline 6 & 1 & 1,6 & 1 & 1,9 & 2 & 7,7 & 1 & 2,6 & - & -..- & 5 & 2,5 \\
\hline 7 & 2 & 3,2 & 1 & 1,9 & - & --- & 2 & 5,1 & 1 & 5,0 & 6 & 3,0 \\
\hline 8 & 1 & 1,6 & 1 & 1,9 & - & -.- & - & $-\cdots$ & - & $\ldots$ & 2 & 1,0 \\
\hline 9 & - & --- & 1 & 1,9 & - & --- & 2 & 5,1 & 1 & 5,0 & 4 & 1,9 \\
\hline 10 & 5 & 7,9 & 5 & 9,2 & 5 & 19,2 & 5 & 12,8 & 3 & 15,0 & 23 & 11,4 \\
\hline TOTAL & 63 & 100,0 & 54 & 100,0 & 26 & 100,0 & 39 & 100,0 & 20 & 100,0 & 202 & 100,0 \\
\hline
\end{tabular}

Fonte: Arquivo da Dermatologia Sanitária - CSE Geraldo Paula Souza - FSP - USP 
TABELA - 18 - Distribuição dos diagnósticos de Cancro Mole realizados no Município de São Paulo - Serviço de Dermatologia Sanitária da Faculdade de Saúde Pública da Universidade de São Paulo, por enfartamento inguinal, 1985 - 1989.

\begin{tabular}{|c|c|c|c|c|c|c|c|c|c|c|c|c|}
\hline \multirow{2}{*}{ ANO } & \multicolumn{2}{|c|}{1985} & \multicolumn{2}{|c|}{1986} & \multicolumn{2}{|c|}{1987} & \multicolumn{2}{|c|}{1988} & \multicolumn{2}{|c|}{1989} & \multicolumn{2}{|c|}{ TOTAL } \\
\hline & № & $\%$ & No & $\%$ & № & $\%$ & № & $\%$ & Ne & $\%$ & № & $\%$ \\
\hline Direito & 11 & 17,5 & 8 & 14,8 & 6 & 23,1 & 4 & 10,3 & 3 & 15,0 & 32 & 15,8 \\
\hline Direito e Esquerdo & 7 & 11,1 & 13 & 24,1 & 1 & 3,9 & 10 & 25,6 & 5 & 25,0 & 36 & 17,8 \\
\hline Ausente & 36 & 57,1 & 25 & 46,3 & 14 & 53,8 & 20 & 51,3 & 8 & 40,0 & 103 & 51,0 \\
\hline TOTAL & 63 & 100,0 & 54 & 100,0 & 26 & 100,0 & 39 & 100,0 & 20 & 100,0 & 202 & 100,0 \\
\hline
\end{tabular}

Fonte: Arquivo da Dermatologia Sanitária - CSE Geraldo Paula Souza - FSP - USP 
Um aspecto importante está relacionado com a associação ou concomitância de doenças. Atualmente é recomendado a feitura do teste sorológico para Sífilis - V.R.D.L. (Veneral Diseases Research Laboratory) em todo o portador de uma DST na tentativa de detectar a Sífilis sorológica inaparente.

$\mathrm{Na}$ Tabela 19 observamos 57 (28,2\%) soros reagentes com Antígenos lipídicos apresentando variação quantitativa de 1 a 128 diluições. Os exames reagentes foram confirmados pelo teste treponêmico FTA abs. Nos surtos de 1945 e 1952 a sorologia para Sífilis variou de 10 a 15\% (MCCARLEY et al, 1988). Na observação africana de NSANZE et al (1981) 11 dos 60 pacientes (18,3\%) e LATIF et al (1986) 4,2\% apresentaram sorologia reagente.

$\mathrm{Na}$ Tabela 20 podemos observar que dos sororeagentes $42,1 \%$, apresentou título maior ou igual a $1 / 16$ diluições, resultado este que reforça a necessidade da feitura do teste treponêmico (FTAabs) confirmatório.

Portanto o percentual detectado nos 202 portadores de Cancro Mole é muito superior ao observado nos surtos americanos e africanos, alertando o observador para a necessidade da sorologia de rotina com a finalidade de detectar possivel associação com Sífilis.

Na Tabela 21 podemos observar que no periodo de 1989 a 1991, 42 portadores de Cancro Mole foram submetidos ao teste para detecção de anticorpos Anti-Vírus da Imunodeficiência Humana-1, (ELISA) resultando em $16,6 \%$ de positividade confirmada após a repetição do teste, pelo Western-Blot. 
O Cancro Mole parece ser importante fator para a aquisição e a transmissão do $\mathrm{VIH}-1$ durante a intercurso sexual (KREISS et al, 1989; WAUGH 1989; FELMAN, 1989) e que portadores de Cancro Mole ou com recente história de úlceras genitais apresentam um maior risco de soropositividade para VIH-1 (RONALD \& PLUMMER - 1989). Em recente observação no nosso meio ALMEIDA et al (1991) observou 9,8\% de soropositividade para VIH-1 em portadores de DST. Na Tabela 22 podemos observar apenas 1 caso feminino positivo. A probabilidade, neste grupo, da mulher adquirir o cancro mole é menor do que a do homem na proporção aproximada de 13 homens para 1 caso feminino.

Apesar da amostra soropositiva e a soronegativa serem quantitativamente diferentes, para o homem não houve diferença significativa entre a presença do Cancro Mole e a soropositividade para o $\mathrm{VIH}-1$, mas para a mulher foi significante, indicando que o risco de uma portadora de cancro mole de se infectar pelo $\mathrm{VIH}-1$ é maior. 
TABELA - 19 - Distribuição dos diagnósticos de Cancro Mole realizados no Município de São Paulo - Serviço de Dermatologia Sanitária da Faculdade de Saúde Pública da Universidade de São Paulo, por resultado qualitativo do VDRL, 1985 - 1989.

\begin{tabular}{|c|c|c|c|c|c|c|c|c|c|c|c|c|}
\hline \multirow[b]{2}{*}{ RESULTADO } & \multicolumn{2}{|c|}{1985} & \multicolumn{2}{|r|}{1986} & \multicolumn{2}{|c|}{1987} & \multicolumn{2}{|c|}{1988} & \multicolumn{2}{|c|}{1989} & \multicolumn{2}{|c|}{ TOTAL } \\
\hline & $\mathrm{N}^{2}$ & $\%$ & No & $\%$ & № & $\%$ & Ne & $\%$ & № & $\%$ & No & $\%$ \\
\hline Reagente & 23 & 36,5 & 16 & 29,6 & 5 & 19,2 & 8 & 20,5 & 5 & 25,0 & 57 & 28,2 \\
\hline Não Reagente & 40 & 63,5 & 38 & 70,4 & 21 & 80,8 & 31 & 79,5 & 15 & 75,0 & 145 & 71,8 \\
\hline TOTAL & 63 & 100,0 & 54 & 100,0 & 26 & 100,0 & 39 & 100,0 & 20 & 100,0 & 202 & 100,0 \\
\hline
\end{tabular}

Fonte: Arquivo da Dermatologia Sanitária - CSE Geraldo Paula Souza - FSP - USP 
TABELA - 20 - Distribuição dos diagnósticos de Cancro Mole realizados no Municipio de São Paulo - Serviço de Dermatologia Sanitária da Faculdade de Saúde Pública da Universidade de São Paulo, por resultado quantitativo do VDRL, 1985 - 1989.

\begin{tabular}{|c|c|c|c|c|c|c|c|c|c|c|c|c|}
\hline \multirow{2}{*}{$\begin{array}{ll} & \text { ANO } \\
\text { RESULTADO } & \\
\end{array}$} & \multicolumn{2}{|c|}{1985} & \multicolumn{2}{|c|}{1986} & \multicolumn{2}{|c|}{1987} & \multicolumn{2}{|c|}{1988} & \multicolumn{2}{|c|}{1989} & \multicolumn{2}{|c|}{ TOTAL } \\
\hline & No & $\%$ & No & $\%$ & № & $\%$ & $\mathrm{~N}^{2}$ & $\%$ & No & $\%$ & $\mathrm{~N}^{\mathrm{O}}$ & $\%$ \\
\hline Não Reagente & 40 & 63,5 & 38 & 70,4 & 21 & 80,9 & 31 & 79,4 & 15 & 75,0 & 145 & 71,8 \\
\hline $1 / 1$ & 3 & 4,8 & 3 & 5,5 & 1 & 3,8 & 3 & 7,6 & 3 & 15,0 & 13 & 6,4 \\
\hline $1 / 2$ & 6 & 9,5 & - & $\cdots$ & - & --- & - & $\cdots$ & 1 & 5,0 & 7 & 3,5 \\
\hline $1 / 4$ & 5 & 7,9 & - & --- & 1 & 3,8 & 1 & 2,6 & - & --- & 7 & 3,5 \\
\hline $1 / 8$ & 4 & 6,3 & 1 & 1,9 & 1 & 3,8 & - & --. & - & ---- & 6 & 3,0 \\
\hline $1 / 16$ & 2 & 3,2 & 3 & 5,5 & 2 & 7,7 & 1 & 2,6 & - & --- & 8 & 3,9 \\
\hline $1 / 32$ & 1 & 1,6 & 3 & 5,5 & - & $\ldots$ & 1 & 2,6 & 1 & 5,0 & 6 & 3,0 \\
\hline $1 / 64$ & 2 & 3,2 & 5 & 9,3 & - & -.- & 1 & 2,6 & - & .... & 8 & 3,9 \\
\hline $1 / 128$ & - & $\ldots$ & 1 & 1,9 & - & $\ldots$ & 1 & 2,6 & - & $-\cdots-$ & 2 & 1,0 \\
\hline TOTAL & 63 & 100,0 & 54 & 100,0 & 26 & 100,0 & 39 & 100,0 & 20 & 100,0 & 202 & 100,0 \\
\hline
\end{tabular}

Fonte: Arquivo da Dermatologia Sanitária - CSE Geraldo Paula Souza - FSP - USP 
TABELA - 21 - Distribuição dos diagnósticos de Cancro Mole realizados no Município de São Paulo - Serviço de Dermatologia Sanitária da Faculdade de Saúde Pública da Universidade de São Paulo, por resultado da deteç̧ão de Anticorpos Anti-HIV-1 (ELISA) e Western-Blot, 1989 - 1991.

\begin{tabular}{|c|rr|rr|c|}
\hline \multirow{2}{*}{ EXAME } & \multicolumn{2}{|c|}{ ELISA } & \multicolumn{2}{|c|}{ WESTERN BLOT } & TOTAL \\
ANO & - & + & - & + & \\
\hline 1989 & 17 & 3 & 17 & 3 & 20 \\
1990 & 13 & 2 & 13 & 2 & 15 \\
1991 & 6 & 1 & 6 & 1 & 7 \\
\hline TOTAL & 36 & 6 & 36 & 6 & 42 \\
\hline
\end{tabular}

Fonte: Laboratório de Dermatologia Sanitária - Departamento de Epidemiologia - FSP - USP 
TABELA - 22 - Distribuição dos diagnósticos de Cancro Mole realizados no Município de São Paulo - Serviço de Dermatologia Sanitária da Faculdade de Saúde Pública da Universidade de São Paulo, por resultado do teste de ELISA e Western-Blot e sexo, 1989 - 1991.

\begin{tabular}{|c|c|c|c|c|c|c|}
\hline $\begin{array}{l}\text { EXAME } \\
\text { SEXO }\end{array}$ & & & e & WEST & BLOT & \\
\hline ANO & M & $\mathrm{F}$ & & $\mathbf{M}$ & F & TOTAL \\
\hline 1989 & 15 & 2 & & 2 & 1 & 20 \\
\hline 1990 & 13 & - & & 2 & - & 15 \\
\hline 1991 & 6 & - & & 1 & - & 7 \\
\hline TOTAL & 34 & & 2 & 5 & 1 & 42 \\
\hline
\end{tabular}

Fonte: Laboratório de Dermatologia Sanitária Departamento de Epidemiologia - FSP - USP 


\section{6 - CONCLUSÕES}

॥

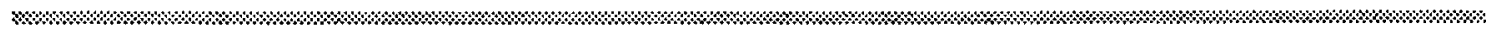




\section{6 - CONCLUSŌES}

Há que, inicialmente, deixar bem claro o que se mencionou anteriormente, ou seja, de que o pretendido neste trabalho vem a ser tão somente fornecer indícios para possíveis estudos epidemiológicos em doença que não é de notificação compulsória e é de letalidade nula.

Posto isso, os dados colhidos apenas revelam o impacto em serviço de assistência ambulatorial e, portanto, as conclusões apresentadas a seguir, poderão nortear futuros inquéritos epidemiológicos em nosso meio.

1 - Houve aumento progressivo dos casos registrados em menores de 20 anos.

2 - A clássica diferença a favor do sexo masculino mostrou tendência a diminuir.

3 - A prostituição ano a ano diminuiu sua participação como fonte de contágio.

4 Os subdistritos do Butantã, Santo Amaro e Sé apresentaram uma maior concentração de casos (55).

5 - O Escritório Regional de Saúde (ERSA-1), concentrou o maior número de casos (74).

6 - A soro reatividade para sífilis $(28,2 \%)$ revelou-se alta.

7 - A soro reatividade para $\mathrm{VIH}-1$ foi de $16,6 \%$.

8 - O risco da mulher portadora de cancro mole se apresentar infectada pelo $\mathrm{VIH}-1$, parece ser maior do que para o homem. 
Diante disso, cabe recomendar a implantação imediata da notificação compulsória de todas as doenças sexualmente transmissiveis, bem como a pesquisa rotineira para sífilis e para vírus da imunodeficiência humana- $f$ nas populações de risco identificadas.

Com as conclusões acima, pensamos haver evidências para sugerir hipóteses a serem objeto de estudos epidemiológicos no Município de São Paulo. 


\section{7 - REFERÊNCIAS BIBLIOGRÁFICAS}


ABECK, D. \& KORTING, H.C. Mechanism of skin adherence, penetration and tissue necrosis production by Haemophilus ducreyi, the causative agent of chancroide. Acta. Venerol., 174(Suppl.):5-20, 1991.

ALMEIDA, R.G.; SIQUEIRA, L.F.G.; SANTOS JUNIOR., M.F.Q.; OYAFUSO, L.K.; LOURENÇO, R.; PIOVESAN, M.; AYROZA-GALVÃO, P.A. Infecção por HIV-1 em portadores de Doenças Sexualmente Transmissíveis (DST) em São Paulo, Brasil. Apresentado ao Congresso Brasileiro de Infectologia - Salvador, Bahia, 27 a 31 de agosto de 1991.

ASIN, J. Chancroid: a report of 1402 cases. Amer. J. Syph., 36:483-7, 1952.

BECKER, T.N.; DEWITT, W.; VAN DUSEN, G. Haemophilus ducreyi infection in south Florida: a rare disease on the rise? South. Med. J., 80:182-4, 1987.

BELDA, W. Cancro mole. In: VERONESI, R. Doenças Infecciosas e Parasitárias. Rio de Janeiro, Ed. Guanabara-Koogan, 1982. p.9902.

BELDA, W.; SIQUEIRA, L.F.G.; BELDA JUNIOR, W.; SANTOS JÚNIOR, M.F.Q. Aspectos atuais do Cancro mole. Bol. Inf. Union, 8(29)3-11, 1983.

BELDA, W.; SANTOS JUNIOR, M.F.Q.; BELDA JUNIOR, W.; SIQUEIRA, L.F.G.; FAGUNDES, L.J.; LOMBARDI, C. Novos rumos no tratamento do Cancro mole - Experimentação cl]inica com Thianphenicol. An. bras. Derm., 59:147-9, 1984. 
BELDA, W.; SANTOS JUNIOR, M.F.Q.; SIQUEIRA, L.F.G.; FAGUNDES, L.J.; BELDA JUNIOR, W.; LOMBARDI, C. Emprego do tianfenicol granulado em dose única de $5,0 \mathrm{~g}$ no tratamento do Cancro mole. An. bras. Derm., 59:209-12, 1984.

BELDA JUNIOR, W.; SANTI, C.G.; MIRANDA, A.A. Tratamento do Cancróide com Tianfenicol. Rev. bras. Med., 42:204-5, 1985.

BLACKMORE, C.A.; LIMPAKARNJANARAT, K.; RIGAU-PEREZ, J.G.; ALBRITTON, W.L.; GREEWOOD, J.R. An outbreak of chancroid in Orange County. California: Descriptive epidemiology and disease control measures. J. infect. Dis., 151:840-4, 1985.

BOGAERTS, J.; RICART, C.A.; VAN DYCK, E.; PIOT, P. The etiology of genital ulceration in Rwanda. Sex. Trans. Dis., 16: 123-6, 1989.

BOPP, C.; MULLER, R.; GERVINI, R.L.; RUFFINI, M.C. Ressurgimento do Cancro Venéreo Simples no Rio Grande do Sul. An. bras. Derm., 48:301-10, 1973.

BORGES, D.R. Cancro mole extra-genital. Arch. derm. syph. S.Paulo, 2:130-136, 1938.

BOUDGHENE-STAMBOULI, O. \& MERAD-BOUDLA, A. Une ulceration genitale "Inhabituelle" en Algérie: le chancro mou. Bull. Soc. Path. Exot., 82:199-200, 1989.

C.D.C. Chancroid. Massachusets. Morb. Mort. Wkly Rep., 34:713-9, 1985.

C.D.C. Sexually Transmitted Disease. Surveillance, 1990. 
CHANCROID, Editorial. Lancet, 2:747-8, 1982.

CASAS, J.G.; ALVAREZ, O.J.; ALVARES, M.I.C. Chancro blando incremento de casos en los momentos actuales. Arch. argent. Derm., 23:118-21, 1973.

CASTRO, B.M. \& ASSIS, L. Cancro venéreo extra-genital. Rev. ass. paul. Med. São Paulo, 10:261-2, 1937.

CHOUINARD, A. Winnipeg suffered second chancroid outbreak, team reports. Can Med. Assoc. J., 140:72-3, 1989.

CIVATTE, J.; BELAICH, S.; BONVALET, D.; VALLET, C.: BROISSIN, M. Réapparition du chancre mou. A propós de l'épidémie parisienne actuelle. Ann. Méd. interne, 7:427-30, 1980.

D'COSTA, L.J.; PLUMMER, F.A.; BOWMER, I.; FRANSEN, L.; PIOT, P.; RONALD, A.R.; NSANZE, H. Prostitutes are a major reservoir of sexually transmitted diseases in Nairobi, Kenya. Sex. transm. Dis., 12:64-7, 1985.

DEGOS, R.; DUPERRAT, B.; GRACIANKY, P. et al. Sur la recrudescence actuelle des chancres mou. Bull. Soc. tranç. Derm. Syph., 80 :5978, 1973.

DUPERRAT, B.; SABLET, M.; BADILLET, G. Épidémie de chancres a Paris. Concours. Med., 95:7063-5, 1973.

FELMAN, Y.M. Recent developments in sexually transmitted disease: Chancroid - epidemiology diagnosis and treatment. Cutis, 44:113-4, 1989. 
FORATTINI, O.P. Ecologia, Epidemilogia e Sociedade. São Paulo, Liv. Ed. Artes Médicas/Ed. Universidade de São Paulo, 1992.

GAY PRIETO, S. Dermatologia, Editorial Científico Médica. 1966.

GREENBLATT, R.M.; LUKEHART, S.A.; PLUMMER, F.A. Genital ulceration as a risk factor for human immunodeficiency virus infection in Kenia. AIDS, 2:47-50, 1988.

HAFIZ, S.; KINGHORN, G.R.; McENTERGART, M.G. Chancroid in Sheffield: a report of 22 cases diagnosed by isolating Haemophilus ducreyi in a modified medium. Brit. J. vener. Dis., 57:382-6, 1981.

HAMMOND. G.W.; SLUTCHUK, M; SCATLIFF, J.; SHERMAN, E., WILT, J.C.; RONALD, A.R. Epidemiologic clinical, laboratoy and therapeutic features of an urban outbreak of chancroid in North America. Rev. Infec. Dis., 2:867-79, 1980.

JESSAMINE, P.G.: SLUTCHAK, M.; BENNIGASN, G.; SHERMAN, E.; BEREZOWSKI, K.; TOYE, B.; BRUMHAN, R.C. Chancroid outbreak Winnipeg Manitoba. Can. Dis. Wkly. Rep., 14:13-4, 1988.

JOSHI, R.M. \& LAWANDE, R.V. Chancroid in Nigeria. Trans. roy. Soc. trop. Med. Hyg. 77:665-7, 1983.

KHOO, R.; SNG, E.H.; GOH, A.J. A study of sexually transmitted diseases in 200 prostitutes in Singapore. Asin J. infect. Dis., 1:77-9, 1977. 
KREISS, J.K.; COOMBS, PLUMMER, F.; HOLMES, K.K.; NIKORA, B.; CAMERON, W.; NGUGI, E.; NDINYA ACHOLA, J.O.; COREY,

L. Isolation of human immunodeficiency virus from genital ulcers in Nairobi Prostitutes. J. infect. Dis., 160:380-4, 1989.

LAO, D.G.; TRUSSELL, R.E. Chancroid in women in Manila. Amer. J. Syph., 31:277-81, 1947.

LATIF, A.S.; CROCCHIOLO, P.R.; LENCIONI, R.. Thiamphenicol in the treatment of chancroid in men. Sex. transm. Dis., 11(suppl. 4):454-5, 1984.

LEME, J.M. Cancro Molle in O Problema Venéreo. (Tese apresentada à Faculdade de Medicina de São Paulo). São Paulo, 1926.

LEPORS, M.J.; PEROL, Y. Identification par cultures du bacile de Ducreyi á l'occasion de deux cas actuels de chancre mou. Bull. Soc. franç. Derm. Syph., 80:598-602, 1973.

LYKKE-OLESEN, L.; LARSEN, L.; PEDERSEN, T.G.; GAARSLEV, K. Epidemic of chancroid in Greenland: 1977-1978. Lancet, 1:654-5, 1979.

MARCKMAN, P.; HOJBJERG, T.; EYBEN, F.E.; CHRISTENSEN, I. Imported pedal chancroid: a case report. Genitourin. Med., 65:126-7, 1989.

MARCHAND, J.P.; STOBEL, M.; N'DIAYE, B. Le chancre mou á Dakar. A propos de 1585 observations. Bull. Soc. med. Afr. noire Langue franç., 23:14-8, 1978. 
MARILL, F.G.; SAYAG, J.: HADIDA, E. Dispariation de la chancrelle en Algerie. Bull. Soc. franç., 23:14-8, 1970.

MAUFF, A.C.; BALLARD, R.C.; BILGERI, Y.R.; KOORNHOFF, H.J. Isolation of Haemophilus ducreyi from genital ulcerations in white men in Johannesburg. S. Afr. med. J., 63:236-7, 1983.

MCCARLEY, M.E.; CRUZ JR.; P.D.; SONTHEIMER, R.D. Chancroid Clinical variants and other findings from an epidemic in Dallas County 1986-1987. J. Am. Acad. Dermatol., 19:330-7, 1988.

MEZZADRA, G.; CHIARENZA, A.; SCUDERI, G. A proposito di una recente epidemia di ulcera venerea osservata nella Sicilia sudorientale. G. Minerva Derm., 112:545-9, 1977.

MINDEL, A. Chancroid epidemics in some developing countries but still rare in Britain. Brit. Med. J., 298:64-5, 1989.

MOREL, P. Le chancre mou. A propos de 55 nouvelles observations. Nouv. Presse Med., 3:1204-6, 1974.

MOREL, P.; CASIN, I.; GANDIOL, C.; VALLET, C.; CIVATTE. Epidémie de chancre mou: traitement de 587 malades. Nouv. Presse Méd., 11:655-6, 1982.

MURAT, A.; OKE, N.; BARANSII, O. Ulcus molle. Epidemie in der Turkie. Hautarzt, 29:573-85, 1978.

NAYYAR, K.C.; STOLZ, E.; MICHEL, M.F. Rising incidence of chancroid in Rotterdan: epidemiological, clinical, diagnostic, and therapeutic aspects. Brit. J. vener. Dis., 55:439-41, 1979. 
NSANZE, H.; FAST, M.V.; D'COSTA, L.J.; TUKEI, P.; CURRAN, J.; RONALD, A. Genital Ulcers in Kenya. Br. J. Vener. Dis., 57:378-81, 1981.

OLIVEIRA, P.P. Cancróide: sua epidemiologia no Exército. Rev. de Med. Militar, 40:37-46, 1951.

ORELLANA-DIAZ, O \& HERNANDEZ-PEREZ, E.. Chancroid in EI Salvador - Increasing incidence, clinical, features and therapeutics. Int. J. Dermatol., 27:243-5, 1988.

PASSOS, M.R.L.; GOULART FILHO, R.A.; SOUZA, E.T.; SOUZA NETTO, B.A. Cancro Mole. Análise terapêutica de 91 casos. Ci. Med. UFF., Niterói, 5/6:69-72, 1987.

PEREIRA JR., A.C. \& SERRUYA, J. Doenças Sexualmente Transmissíveis - Proposta de Classificação. An. bras. Derm., 56:137-8, 1981.

PIOT, P. \& MEHEUS, A. Genital ulceration in the tropics. In: ORIEL, J.D. \& HARRIS, J.R.M. Recent advences in sexually transmitted diseases. Churchill Livingstone Edinburgh, 1966.

PLATteaU, M.; DE MAUBEUGE, J.; ACHTEN, G. Deux cas de chancre mou. Dermatologica, Basel, 174:193-5, 1987.

PLUMMER, F.A.; D'COSTA, L.J.; NSANZE, H.; DYLEWSKI, J.; KARASIRA, P.; RONALD, A.R. Epidemiology of chancroid and Haemophilus ducreyi in Nairobi, Kenya. Lancet, 2:1293-5, 1983. 
PLUMMER, F.A.; D'COSTA, L.J.; NSANZE, H. KARASIRA, P.; MACLEAN, I.W.; PIOT, P.; RONALD, A.R. Clinical and microbiologic studies of genital ulcers in Kenyan women. Sex. transm. Dis., 12:193-7, 1985.

PLUMMER, F.A.; WAINBERG, M.A.; PLOURDE, P.; JeSSAMINE, P.; D'COSTA, L.J.; WAMOLA, I.A.; RONALD, A.R. Detection of human immunodeficiency virus type 1 (HIV-1) of in genital ulcer exudate of HIV-1, infected men by culture and gene amplification. J. Infect. Dis., 161:810-811, 1990.

RABELO, F.E. Nomenclatura Epidemiológica. Rio de Janeiro, 1980.

RICORD, P. Traté pratique des maladies vénériennes. Librarie des sciences medicales. Paris, 1838.

RONALD, A.R.; PLUMMER, F. Chancroid. A newly important sexually transmitted disease (editorial). Arch. Derm., 125:1413-4, 1989.

RUDOLPH, A.H. Chancroid. In: FITZPATRICK, B.T. et al. Dermatology in general medicine, 3 ed., 2452-7, 1987.

SABLET, M.; PUISSANT, A. Le chancre mou: se reapparition a Paris, ses complications, son traitement. Prog. Méd., Paris, 102:343-8, 1974.

SAMPAIO, S.A.P. Estatísticas sobre freqüência de sífilis e moléstias venéreas em São Paulo. Rev. paul. Med., 51:113-8, 1957.

SANCHEZ, M.A.; VERA, J.R.M.; CANAS, J.M.E.; LOURIDO, F.B. Chancro blando: dos casos con cultivo positivo. Actas DermoSif., 80:230-4, 1989. 
SANTOS JUNIOR, M.F.Q. \& BELDA, W. Porque e o que fazer no controle e profilaxcia das D.S.T. Ars. Curandi, 19:113-20, 1986.

SATULSKY, E.M. Management of chancroid in a tropical theatre. Report of 1555 cases. J. Amer. med. Ass., 127:259-63, 1945.

SCHMID, G.P.; SANDERS, L.L.; BLOUNT, J.H.; ALEXANDER, R. Chancroid in the United States reestablishment of an old disease. J. Amer. med. Ass., 258:3265-8, 1987.

SEKKAT, A.; ROLLIER, R.; BENHAYOUNE, T.S. et al. The soft chancre and its treatment (781 patients). Maroc. med., 6:237-47, 1984.

SERRUYA, J. \& ALMEIDA, B.B. Cancro mole. Rev. Med., 7:31, 1977.

SIBOULET, A. Maladies Sexuellement Transmissibles. Paris, MASSON, 1975.

SIMONS, R.D.G. Clinical features in over 1200 syphilitic chancres and chancroid and over 140 cases of lymphopathia venerea. Acta derm. vernereol., 27:115-22, 1947.

SMITH, G. \& DOBSON, A.P. Sexually transmitted diseases in animals. Parasitology Today, 8:159-166, 1992.

SPARLING, P.G. Sexually transmitted diseases. In: Wyngaarden, J.B.; Smith, L.H.: Bennett, J.C., eds. Cecil textbook of medicine, 2 vol. Philadelphia, W.B. Saunders Co., 1992.

STRAKOSCH, E.A.; KENDAL, H.W.; CRAIG, R.M.; SCHWEMLEIN, G.X. Clinical and laboratory investigation of $\mathbf{3 7 0}$ cases of chancroid. J. invest. Derm., 6:95-107, 1945. 
STURM, A.W.; STOLTING, G.J.; CORMANE, R.H.; ZANEN, H.C. Clinical and microbiological evaluation of 46 episodes of genital ulceration. Genitourin. Med., 63:98-101, 1987.

TAN, T., RAJAN, V.S.; KOE, S.H.; TAN, N.J.; TAN, B.H.; GOH, A.J. Chancroid a study of 500 cases. Asian J. infect. Dis., 1:27-8, 1977.

TAYLOR, D.N.; DUANGMANI, C.; SUVONGSE, C.; O'CONNOR, R.; PITARANGSI, C.; PANIKABUTRA, K.; ECHEVERRIA, P. The role of Haemophilus ducreyi in penile ulcers in Bangkok, Thailand. Sex. Transm. Dis., 11:148-51, 1984.

TEIXEIRA, L.A.N.; ALMEIDA, J.L.M. Ensaio terapêutico com o emprego do tianfenicol no tratamento do Cancro mole e da blenorragia. A Folha Médica, 90:359-60, 1985.

W.H.O. WHO Features ne 152, 1990.

WAUGH, M.A. Chancroid and HIV. Br. Med. J., 298:321, 1989. 